IMA Journal of Applied Mathematics (2005) 1-25

doi: 10.1093/imamat/hxh000

\title{
Instability in the self-similar motion of a planar solidification front
}

\author{
Ferran Brosa Planella, Colin P. Please and Robert A. Van Gorder* \\ Mathematical Institute, University of Oxford, Andrew Wiles Building, Radcliffe \\ Observatory Quarter, Woodstock Road, Oxford OX2 6GG United Kingdom
}

[Received on xx; accepted on $\mathrm{xx}$ ]

\begin{abstract}
Understanding the solidification process of a binary alloy is important if one is to control the microstructure obtained during the casting of metals. While much work has been done on the steady state solidification problem, despite their relevance to metallurgical applications, there is less known about non-steady solidification problems and their stability. In the paper we shall consider the non-steady solidification problem in which the planar solidification front moves in a self-similar manner, in both infinite and semi-infinite planar one-dimensional geometries. For each geometry exact solutions are known for the resulting Stefan problem. We direct our attention to the stability of each solution, demonstrating that while the concentration and thermal solutions remain stable, the interface corresponding to the solidification front can develop instabilities. For each geometry, we find that there are always unstable perturbations, although we observe qualitative differences in the form of the unstable perturbations for each case. These results generalize and extend several existing studies in the literature, and throw light on the instability inherent in the non-steady solidification process.
\end{abstract}

Keywords: solidification front, directional solidification, stability analysis

\section{Introduction}

Understanding the microstructure of a metal alloy is crucial to controlling the quality of a cast in many metallurgical applications. One parameter that characterises the microstructure is the grain size, which has a strong dependence on the cooling rate. Higher cooling rates result in smaller grains and slower cooling rates in larger grains. For the application of interest here, metallurgical grade silicon, smaller grains imply a better impurity distribution, which is desirable, but simultaneously the losses due to small particles, created during crushing, gives a lower silicon yield. In Figure 1 we can observe the typical structure of silicon grains. More details on silicon microstructure can be found in Schei et al. (1998); Anglézio et al. (1990); Møll (2014). Knowledge of the dynamics that create the structure will lead to a more effective casting process.

The solidification process can be modelled as a Stefan problem (see Rubinstein (1971); Worster (2002) for details), and it has been shown in Benham et al. (2016) that even relatively simple models can accurately predict the temperature profile and the solidification front position in a silicon cast in a wedge-shaped mould cooled by the contact with the atmosphere. There

*Email: Robert.VanGorder@maths.ox.ac.uk 


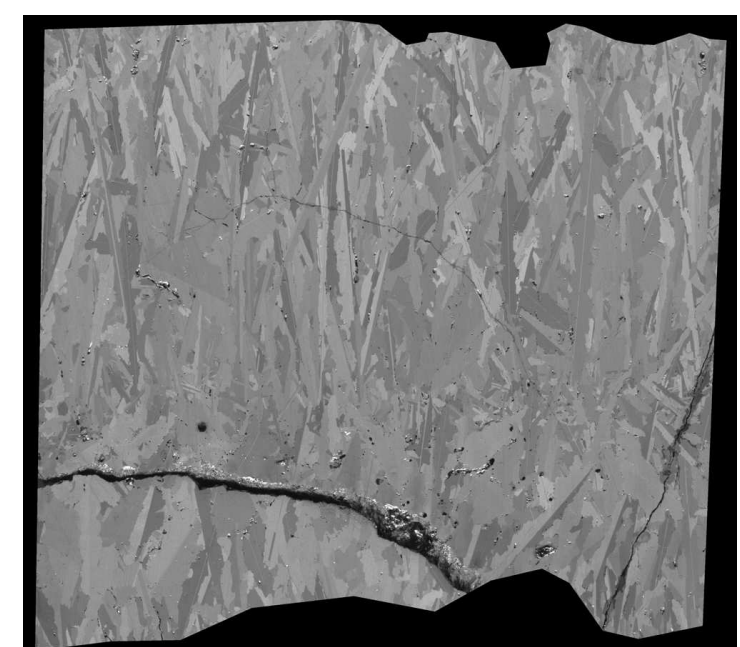

FIG. 1: Macroscopic picture of a silicon sample, taken from an experiment at Elkem where the sample was cooled from the lower boundary. We can observe different grain sizes: smaller near the lower boundary and larger near the upper boundary.

has been a lot of work done on the directional solidification process, where the velocity of the solidification front is constant and known (so the problem can be reduced to a steady state problem). The stability analysis for the steady solidification binary alloy problem was first considered by Mullins and Sekerka (1964), who consider the 2D problem in an infinite domain under infinitesimal sinusoidal perturbations. By assuming that the velocity of the solidification front is both constant and known they obtain a steady state base solution, and find that the phenomenon that causes the instability in the problem is the constitutional supercooling. The same analysis was extended by Wollkind and Segel (1970) to perturbations on a finite domain. The main difference between their analysis and that in Mullins and Sekerka (1964) is that, for some parameter configurations, there is a subcritical instability and a non-trivial equilibrium point that was not predicted in Mullins and Sekerka (1964). This type of analysis has proven useful for modelling directional solidification, since in this case the velocity of the solidification front is controlled. However, for the casting method of interest here, casting in a mould with no control on the heat exchange, the assumption of constant velocity for the solidification front is no longer reasonable. Instead, we expect a self-similar motion, as suggested by Benham et al. (2016). Aside from the findings in Benham et al. (2016), the necessity of studying solidification problems involving self-similar solidification front motion was highlighted by Langer (1980). Therefore, it is this type of solidification which we shall focus our efforts on here.

The stability analysis for the non-steady solidification problem, in particular the problem of the self-similar motion of a solidification front, was first studied by Coriell et al. (1999), where exact 1D self-similar solutions were obtained in an infinite domain and a stability analysis was performed. The stability analysis presented addresses a particular type of possible perturbation in terms of parabolic cylinder functions (a more general stability analysis will be considered in the present paper). The analysis was extended to 2D by Alexandrov in Alexandrov (2001, 2004). The analysis in both papers is based on the 1D stability analysis described in Coriell et al. 
(1999), so the perturbations considered are not the most general ones possible. In Alexandrov (2001) there are no assumptions made on the conditions for the transverse coordinate, whereas in Alexandrov (2004) the assumption that the perturbations are linear in the transverse direction is taken. The stability of the 2D problem in a semi-infinite domain has been considered as well in Lambert and Rangel (2002) using a frozen-time approach. This approach allows the authors to find the wavelength of the $2 \mathrm{D}$ perturbations but it does not match the observed grain size well.

In this paper, we perform a comprehensive stability analysis for the 1D binary alloy solidification problem due to self-similar motion of a planar solidification front. In Section 2 we present the exact similarity solutions in both the infinite and semi-infinite domains. In Section 3 we consider the general linear stability problem, obtaining a more general form of perturbation than used in Coriell et al. (1999); Alexandrov (2001, 2004). We apply this stability analysis to the semi-infinite domain problem (which has not been considered in the literature) in Section 4, and we find that the system is unstable for any parameter range. We obtain analogous results for the infinite domain problem (thereby generalising results in Coriell et al. (1999)) in Section 5. We give a discussion of these results in Section 6 .

\section{Self-similar motion of a solidification front}

Even though silicon solidifies into a granular structure, the grains are long and thin and they grow aligned in the direction of the solidification front. It can be shown that any effect in a direction parallel to the solidification front is very small and can be neglected. Therefore we can consider a solidification model with a flat interface, resulting in a 1D problem, as has been considered previously. There are two models we can consider: one on a semi-infinite domain, and one on an infinite domain. The semi-infinite geometry corresponds to a semi-infinite block of initially molten silicon at a constant temperature that is cooled from a wall which is held at a fixed temperature. The wall acts as a heat sink but a barrier for the impurities. The infinite geometry models two semi-infinite blocks of silicon, one initially solid and the other initially liquid, both at a constant temperature. The blocks are then put together and evolve in time. In this problem, there is no heat sink, so the energy of the silicon is conserved. The semi-infinite problem is closest to the physical casting problem, but the infinite geometry gives insight into more general situations.

We model the concentration and temperature fields both in the liquid and the solid phase and we consider any impurities together in a single concentration field. The interface that separates the phases is assumed to be sharp and we denote it by the curve $\hat{z}=\hat{S}(\hat{t})$ in the $\hat{z}-\hat{t}$ plane. In our model we include constitutional supercooling effects and latent heat of fusion. For the supercooling we will assume a simplified phase diagram where the supercooling depends linearly on the concentration of impurities. In our model we allow the densities of the liquid and solid phase to be different, but for simplicity we will not consider the advection caused by the change of density during solidification.

For the solid phase, which is given by $\hat{z}<\hat{S}(\hat{t})$, the equations read

$$
\frac{\partial \hat{c}_{s}}{\partial \hat{t}}=D_{s} \frac{\partial^{2} \hat{c}_{s}}{\partial \hat{z}^{2}} \quad \text { and } \quad \frac{\partial \hat{T}_{s}}{\partial \hat{t}}=\kappa_{s} \frac{\partial^{2} \hat{T}_{s}}{\partial \hat{z}^{2}}
$$

where $\hat{c}_{s}$ is the concentration of impurities and $\hat{T}_{s}$ is the temperature. The liquid phase is given 
by $\hat{z}>\hat{S}(\hat{t})$, and the equations in this region are

$$
\frac{\partial \hat{c}_{l}}{\partial \hat{t}}=D_{l} \frac{\partial^{2} \hat{c}_{l}}{\partial \hat{z}^{2}} \quad \text { and } \quad \frac{\partial \hat{T}_{l}}{\partial \hat{t}}=\kappa_{l} \frac{\partial^{2} \hat{T}_{l}}{\partial \hat{z}^{2}}
$$

where $\hat{c}_{l}$ is the concentration of impurities and $\hat{T}_{l}$ is the temperature in this region. The conditions at the interface $\hat{z}=\hat{S}(\hat{t})$ reduce to

$$
\begin{aligned}
\hat{T}_{s} & =\hat{T}_{l}, \\
\hat{c}_{s} & =-\alpha_{s}\left(\hat{T}_{s}-\hat{T}_{m 0}\right), \\
\hat{c}_{l} & =-\alpha_{l}\left(\hat{T}_{l}-\hat{T}_{m 0}\right), \\
\rho_{s} L \frac{\mathrm{d} \hat{S}}{\mathrm{~d} \hat{t}} & =k_{s} \frac{\partial \hat{T}_{s}}{\partial \hat{z}}-k_{l} \frac{\partial \hat{T}_{l}}{\partial \hat{z}}, \\
\left(\hat{c}_{l}-\hat{c}_{s}\right) \frac{\mathrm{d} \hat{S}}{\mathrm{~d} \hat{t}} & =D_{s} \frac{\partial \hat{c}_{s}}{\partial \hat{z}}-D_{l} \frac{\partial \hat{c}_{l}}{\partial \hat{z}} .
\end{aligned}
$$

The parameters $\kappa_{s}$ and $\kappa_{l}$ are the thermal diffusivities in the solid and liquid phase and they are defined as $\kappa_{j}=k_{j} /\left(\rho_{j} c_{p j}\right)$, where $k_{j}$ is the thermal conductivity, $\rho_{j}$ the density and $c_{p j}$ the heat capacity in the corresponding phase. Also, $D_{j}$ is the diffusivity of impurities, $\alpha_{j}$ is the slope of the phase diagram, and $L$ is the latent heat of solidification.

Because we will seek self-similar solutions, the initial and boundary conditions will be set in a way they satisfy the similarity condition. For the semi-infinite domain problem, we have a mould wall at $\hat{z}=0$. At this wall we set a fixed temperature and a no flux condition for the impurity concentration while far from the interface the liquid remains unchanged so

$$
\begin{array}{r}
\frac{\partial \hat{c}_{s}}{\partial \hat{z}}=0, \quad \text { and } \quad \hat{T}_{s}=\hat{T}_{c}, \quad \text { when } \hat{z}=0, \\
\hat{c}_{l} \rightarrow \hat{c}_{l \infty}, \quad \text { and } \quad \hat{T}_{l} \rightarrow \hat{T}_{l \infty}, \quad \text { when } \hat{z} \rightarrow+\infty .
\end{array}
$$

and we impose the initial data

$$
\hat{c}_{l}=\hat{c}_{l \infty}, \quad \hat{T}_{l}=\hat{T}_{l \infty}, \quad \text { and } \quad \hat{S}=0, \quad \text { when } \hat{t}=0 .
$$

For the infinite domain problem we prescribe far field values for both concentration and temperature fields so

$$
\begin{aligned}
\hat{c}_{s} & \rightarrow \hat{c}_{s \infty}, \quad \text { and } \quad \hat{T}_{s} \rightarrow \hat{T}_{s \infty} & \text { when } \hat{z} \rightarrow-\infty, \\
\hat{c}_{l} & \rightarrow \hat{c}_{l \infty}, \quad \text { and } \quad \hat{T}_{l} \rightarrow \hat{T}_{l \infty} & \text { when } \hat{z} \rightarrow+\infty,
\end{aligned}
$$

and we impose the initial data

$$
\begin{array}{r}
\hat{c}_{s}=\hat{c}_{s \infty}, \quad \hat{T}_{s}=\hat{T}_{s \infty}, \quad \hat{c}_{l}=\hat{c}_{l \infty}, \\
\hat{T}_{l}=\hat{T}_{l \infty}, \quad \text { and } \quad \hat{S}=0, \quad \text { when } \hat{t}=0 .
\end{array}
$$




\subsection{Non-dimensional model}

We now proceed to non-dimensionalise the problem. We take the scalings $\hat{t}=\left(Z^{2} / D_{l}\right) t, \hat{z}=Z z$, $\hat{S}=Z S, \hat{c}=\Delta c c, \hat{T}=\Delta T T+\hat{T}_{m 0}$, where $Z$ is the scaling of the spatial coordinate, $\Delta T$ the scaling of temperature, $\hat{T}_{m 0}$ the melting temperature for pure silicon, and $\Delta c$ is the scaling of concentration. The problem we are studying is self-similar, so we do not need to pick a value for the scaling $Z$. However, a typical choice would be the thickness of the molten silicon layer. With these scalings we arrive at the dimensionless parameters of use, and we define the: Stefan number St $=\Delta T c_{p l} / L$, density ratio $\rho=\rho_{s} / \rho_{l}$, heat conductivity ratio $k=k_{s} / k_{l}$, mass diffusivity ratio $D=D_{s} / D_{l}$, Lewis number Le $=\kappa_{l} / D_{l}$, specific heat ratio $c_{p}=c_{p s} / c_{p l}$, heat diffusivity ratio $\kappa=\kappa_{s} / \kappa_{l}$, slope of the solidus line $m_{s}=\alpha_{s} \Delta T / \Delta c$, slope of the liquidus line $m_{l}=\alpha_{l} \Delta T / \Delta c$, and segregation coefficient $\alpha=\alpha_{s} / \alpha_{l}$.

The dimensionless model is as follows. In the solid phase, defined by $0<z<S(t)$, the governing equations are

$$
\frac{\partial c_{s}}{\partial t}=D \frac{\partial^{2} c_{s}}{\partial z^{2}} \quad \text { and } \quad \frac{\partial T_{s}}{\partial t}=\operatorname{Le} \kappa \frac{\partial^{2} T_{s}}{\partial z^{2}}
$$

the liquid phase is defined by $z>S(t)$, and the equations are

$$
\frac{\partial c_{l}}{\partial t}=\frac{\partial^{2} c_{l}}{\partial z^{2}} \quad \text { and } \quad \frac{\partial T_{l}}{\partial t}=\operatorname{Le} \frac{\partial^{2} T_{l}}{\partial z^{2}}
$$

and the conditions at the interface $z=S(t)$ are

$$
\begin{aligned}
T_{s} & =T_{l}=T_{i}, \\
c_{s} & =-m_{s} T_{i}=\alpha c_{i}, \\
c_{l} & =-m_{l} T_{i}=c_{i}, \\
\frac{\rho}{\operatorname{StLe}} \frac{\mathrm{d} S}{\mathrm{~d} t} & =k \frac{\partial T_{s}}{\partial z}-\frac{\partial T_{l}}{\partial z}, \\
\left(c_{l}-c_{s}\right) \frac{\mathrm{d} S}{\mathrm{~d} t} & =D \frac{\partial c_{s}}{\partial z}-\frac{\partial c_{l}}{\partial z} .
\end{aligned}
$$

The remaining boundary conditions will depend on the problem geometry, and we shall list these later when considering each of the semi-infinite and infinite problems.

In both problems we will seek self-similar solutions of the form $f(\eta)$ where the similarity variable is

$$
\eta=\frac{z}{2 \sqrt{t}}
$$

so the position of the interface will be at $\eta=\lambda$, where $\lambda$ is a constant.

\subsection{The semi-infinite domain problem}

First consider the semi-infinite domain problem. A sketch of this problem is shown in Figure 2. For this problem we have the nondimensional boundary conditions

$$
\begin{gathered}
\frac{\partial c_{s}}{\partial z}=0, \quad \text { and } \quad T_{s}=-1, \quad \text { when } z=0, \\
c_{l} \rightarrow 1, \quad \text { and } \quad T_{l} \rightarrow T_{l \infty}, \quad \text { when } z \rightarrow+\infty,
\end{gathered}
$$



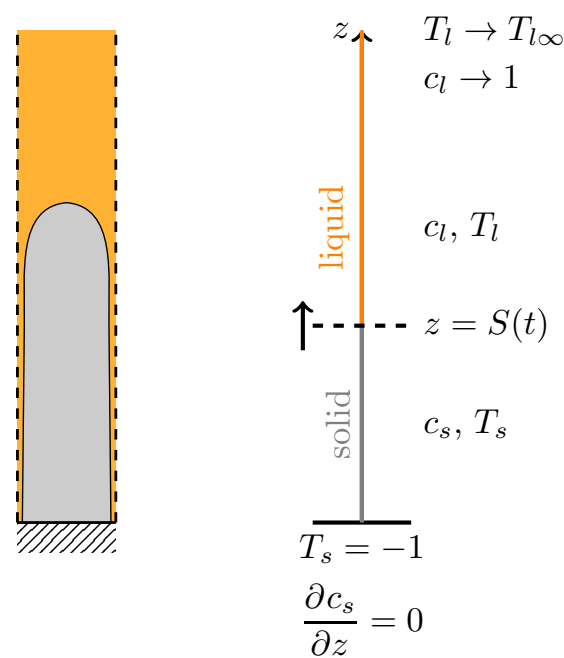

FIG. 2: Sketch of the semi-infinite domain. The diagram on the left shows the original problem we want to solve. Because the dependence on the transverse direction is weak, we can assume that the interface is planar, and hence the model can be reduced to the $1 \mathrm{D}$ model shown in the sketch on the right.

and the initial data

$$
c_{l}=1, \quad T_{l}=T_{l \infty}, \quad \text { and } \quad S=0, \quad \text { when } t=0,
$$

where $T_{l \infty}=\left(\hat{T}_{l \infty}-\hat{T}_{m 0}\right) / \Delta T$. We have chosen $\Delta T=\hat{T}_{m 0}-\hat{T}_{c}$ and $\Delta c=\hat{c}_{l \infty}$ for convenience. The solution for the semi-infinite domain is well known and we write it as

$$
\begin{aligned}
& c_{s}(\eta)=\alpha c_{i} \\
& c_{l}(\eta)=1+\left(c_{i}-1\right) \frac{\operatorname{erfc}(\eta)}{\operatorname{erfc}(\lambda)} \\
& T_{s}(\eta)=-1+\left(T_{i}+1\right) \frac{\operatorname{erf}\left(\frac{\eta}{\sqrt{\kappa \mathrm{Le}}}\right)}{\operatorname{erf}\left(\frac{\lambda}{\sqrt{\kappa \mathrm{Le}}}\right)} \\
& T_{l}(\eta)=T_{l \infty}+\left(T_{i}-T_{l \infty}\right) \frac{\operatorname{erfc}\left(\frac{\eta}{\sqrt{\mathrm{Le}}}\right)}{\operatorname{erfc}\left(\frac{\lambda}{\sqrt{\mathrm{Le}}}\right)} \\
& S(t)=2 \lambda \sqrt{t},
\end{aligned}
$$

where $c_{i}$ and $T_{i}$ are the interface values and $\lambda$ is a constant to be determined. Defining the functions

$$
F(\zeta)=\sqrt{\pi} \zeta e^{\zeta^{2}} \operatorname{erfc}(\zeta) \text { and } \quad G(\zeta)=\sqrt{\pi} \zeta e^{\zeta^{2}} \operatorname{erf}(\zeta)
$$




\begin{tabular}{|c|c|}
\hline Parameter & Value \\
\hline \hline $\mathrm{St}$ & 0.77 \\
$\mathrm{Le}$ & 134 \\
$\rho$ & 0.95 \\
$c_{p}$ & 1 \\
$k$ & 2.91 \\
$\kappa$ & 3.06 \\
$m_{s}$ & 0.69 \\
$m_{l}$ & 346 \\
$D$ & $1.76 \cdot 10^{-2}$ \\
$\alpha$ & $2 \cdot 10^{-3}$ \\
$T_{l \infty}$ & 0 \\
$c_{s \infty}$ & 0 \\
\hline
\end{tabular}

Table 1: Non-dimensional parameter values for silicon. The values shown above have been used in the plots shown later in the article.

the interface values are given by

$$
c_{i}=\frac{1}{1-(1-\alpha) F(\lambda)}
$$

and

$$
T_{i}=\frac{\rho F\left(\frac{\lambda}{\sqrt{\mathrm{Le}}}\right)\left(G\left(\frac{\lambda}{\sqrt{\kappa \mathrm{Le}}}\right)-c_{p} \mathrm{St}\right)+\operatorname{St} T_{l \infty} G\left(\frac{\lambda}{\sqrt{\kappa \mathrm{Le}}}\right)}{\operatorname{St}\left(c_{p} \rho F\left(\frac{\lambda}{\sqrt{\mathrm{Le}}}\right)+G\left(\frac{\lambda}{\sqrt{\kappa \mathrm{Le}}}\right)\right)},
$$

which results in $\lambda$ being given by the transcendental equation

$$
\frac{1}{1-(1-\alpha) F(\lambda)}=-m_{l} \frac{\rho F\left(\frac{\lambda}{\sqrt{\mathrm{Le}}}\right)\left(G\left(\frac{\lambda}{\sqrt{\kappa \mathrm{Le}}}\right)-c_{p} \mathrm{St}\right)+\operatorname{St} T_{l \infty} G\left(\frac{\lambda}{\sqrt{\kappa \mathrm{Le}}}\right)}{\operatorname{St}\left(c_{p} \rho F\left(\frac{\lambda}{\sqrt{\mathrm{Le}}}\right)+G\left(\frac{\lambda}{\sqrt{\kappa \mathrm{Le}}}\right)\right)} .
$$

While the concentration and temperature fields admit closed-form solutions, unless limiting cases of parameters are considered (9) needs to be solved numerically in order to find the value of the parameter $\lambda$. It is not obvious if the value of $\lambda$ is unique. For our parameter choice (see Table 1) we find that there are two possible solutions to (9) but one of them is negative and therefore is not physical.

\subsection{The infinite domain problem}

Now consider the infinite domain problem. We have assumed that there is no heat loss at infinity so the energy of the system is conserved. A sketch of this problem is shown in Figure 3.

For this problem we have the nondimensional boundary conditions

$$
\begin{gathered}
c_{s} \rightarrow c_{s \infty}, \quad \text { and } \quad T_{s} \rightarrow-1, \quad \text { when } z \rightarrow-\infty, \\
c_{l} \rightarrow 1, \quad \text { and } \quad T_{l} \rightarrow T_{l \infty}, \quad \text { when } z \rightarrow+\infty,
\end{gathered}
$$



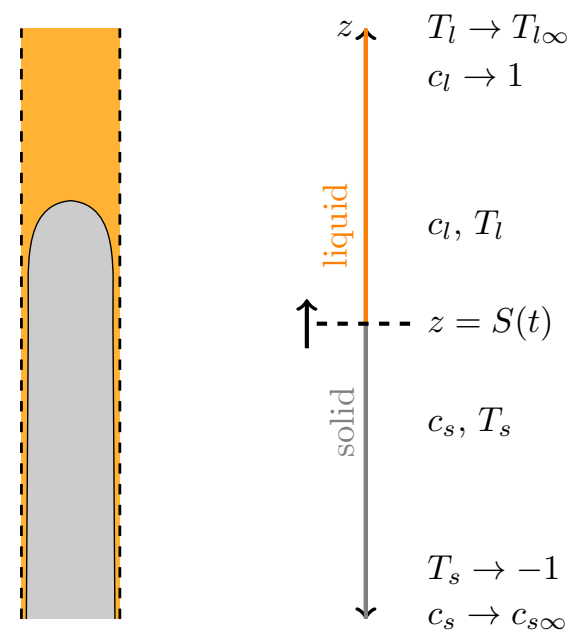

FIG. 3: Sketch of the infinite domain model. The diagram on the left shows the original problem we want to solve. Since the dependence on the transverse direction is assumed to be weak, we take the interface to be planar so that the model can be reduced to a 1D model, as shown in the sketch on the right.

with the initial data

$$
\begin{array}{r}
c_{s}=c_{s \infty}, \quad T_{s}=-1, \quad c_{l}=1, \\
T_{l}=T_{l \infty}, \quad \text { and } \quad S=0, \quad \text { when } t=0,
\end{array}
$$

where $c_{s \infty}=\hat{c}_{s \infty} / \Delta c$ and $T_{l \infty}=\left(\hat{T}_{l \infty}-\hat{T}_{m 0}\right) / \Delta T$ are dimensionless quantities. In this case we have chosen $\Delta T=\hat{T}_{m 0}-\hat{T}_{s \infty}$ and $\Delta c=\hat{c}_{l \infty}$ for convenience. The solution for the infinite domain problem is well known and we write it as

$$
\begin{aligned}
& c_{s}(\eta)=c_{s \infty}+\left(\alpha c_{i}-c_{s \infty}\right) \frac{\operatorname{erfc}\left(-\frac{\eta}{\sqrt{D}}\right)}{\operatorname{erfc}\left(-\frac{\lambda}{\sqrt{D}}\right)}, \\
& c_{l}(\eta)=1+\left(c_{i}-1\right) \frac{\operatorname{erfc}(\eta)}{\operatorname{erfc}(\lambda)}, \\
& T_{s}(\eta)=-1+\left(T_{i}+1\right) \frac{\operatorname{erfc}\left(-\frac{\eta}{\sqrt{\kappa \mathrm{Le}}}\right)}{\operatorname{erfc}\left(-\frac{\lambda}{\sqrt{\kappa \mathrm{Le}}}\right)}, \\
& T_{l}(\eta)=T_{l \infty}+\left(T_{i}-T_{l \infty}\right) \frac{\operatorname{erfc}\left(\frac{\eta}{\sqrt{\mathrm{Le}}}\right)}{\operatorname{erfc}\left(\frac{\lambda}{\sqrt{\mathrm{Le}}}\right)}, \\
& S(t)=2 \lambda \sqrt{t},
\end{aligned}
$$

where $c_{i}$ and $T_{i}$ are the interface values and $\lambda$ is a constant to be determined. The interface 
values are given by

$$
\begin{gathered}
c_{i}=\frac{F\left(-\frac{\lambda}{\sqrt{D}}\right)-c_{s \infty} F(\lambda)}{F\left(-\frac{\lambda}{\sqrt{D}}\right)-F(\lambda)\left[(1-\alpha) F\left(-\frac{\lambda}{\sqrt{D}}\right)+\alpha\right]}, \\
T_{i}=\frac{\rho F\left(\frac{\lambda}{\sqrt{\mathrm{Le}}}\right)\left(-F\left(-\frac{\lambda}{\sqrt{\kappa \mathrm{Le}}}\right)-c_{p} \mathrm{St}\right)-\mathrm{St} T_{l \infty} F\left(-\frac{\lambda}{\sqrt{\kappa \mathrm{Le}}}\right)}{\operatorname{St}\left(c_{p} \rho F\left(\frac{\lambda}{\sqrt{\mathrm{Le}}}\right)-F\left(-\frac{\lambda}{\sqrt{\kappa \mathrm{Le}}}\right)\right)},
\end{gathered}
$$

and hence $\lambda$ is a solution to the transcendental equation

$$
\begin{gathered}
\frac{F\left(-\frac{\lambda}{\sqrt{D}}\right)-c_{s \infty} F(\lambda)}{F\left(-\frac{\lambda}{\sqrt{D}}\right)-F(\lambda)\left[(1-\alpha) F\left(-\frac{\lambda}{\sqrt{D}}\right)+\alpha\right]} \\
=-m_{l} \frac{\rho F\left(\frac{\lambda}{\sqrt{\mathrm{Le}}}\right)\left(-F\left(-\frac{\lambda}{\sqrt{\kappa \mathrm{Le}}}\right)-c_{p} \mathrm{St}\right)-\mathrm{St} T_{l \infty} F\left(-\frac{\lambda}{\sqrt{\kappa \mathrm{Le}}}\right)}{\mathrm{St}\left(c_{p} \rho F\left(\frac{\lambda}{\sqrt{\mathrm{Le}}}\right)-F\left(-\frac{\lambda}{\sqrt{\kappa \mathrm{Le}}}\right)\right)} .
\end{gathered}
$$

Again, it is not obvious if the value of $\lambda$ is unique. For our parameter choice (see Table 1) we find that the solution to (12) is unique.

Now that we have the exact solution to the Stefan problem governing solidification due to the self-similar motion of a planar solidification front in both geometries in the remainder of this paper we shall concentrate on the stability of these solutions to one-dimensional perturbations.

\section{The linear stability problem}

In order to perform a stability analysis for the solidification of a binary alloy, we introduce the perturbed solutions

$$
\begin{aligned}
c_{s, \text { pert }} & =c_{s 0}(t, z)+\delta c_{s 1}(t, z), \\
c_{l, \text { pert }} & =c_{l 0}(t, z)+\delta c_{l 1}(t, z), \\
T_{s, \text { pert }} & =T_{s 0}(t, z)+\delta T_{s 1}(t, z), \\
T_{l, \text { pert }} & =T_{l 0}(t, z)+\delta T_{l 1}(t, z), \\
S_{\text {pert }} & =S_{0}(t)+\delta S_{1}(t),
\end{aligned}
$$

where $c_{s 0}, c_{l 0}, T_{s 0}, T_{l 0}$, and $S_{0}$ are the base state solutions determined in Section 2 . We assume $0<\delta \ll 1$ is a small parameter which measures the size of the perturbation. We will say that the system is unstable if any of the perturbations grows unboundedly in time. Notice that the interface position itself grows unboundedly in time so we will consider the absolute size of $S_{1}$, not the relative size of $S_{1}$ to the base state $S_{0}$, for the stability criteria.

To evaluate the temperature and concentration fields at the perturbed interface, we expand for small $\delta$. For example, the perturbation for the concentration field in the solid will give

$$
c_{s, p e r t}\left(t, S_{\text {pert }}\right)=c_{s 0}\left(t, S_{0}\right)+\delta S_{1} \frac{\partial c_{s 0}}{\partial z}\left(t, S_{0}\right)+\delta c_{s 1}\left(t, S_{0}\right)+\mathcal{O}\left(\delta^{2}\right)
$$


As we are interested in the linear stability problem, we do not consider the terms of $\mathcal{O}\left(\delta^{2}\right)$.

The first order perturbation equations are

$$
\begin{gathered}
\frac{\partial c_{s 1}}{\partial t}=D \frac{\partial^{2} c_{s 1}}{\partial z^{2}} \quad \text { and } \quad \frac{\partial T_{s 1}}{\partial t}=\kappa \operatorname{Le} \frac{\partial^{2} T_{s 1}}{\partial z^{2}} \\
\frac{\partial c_{l 1}}{\partial t}=\frac{\partial^{2} c_{l 1}}{\partial z^{2}} \quad \text { and } \quad \frac{\partial T_{l 1}}{\partial t}=\operatorname{Le} \frac{\partial^{2} T_{l 1}}{\partial z^{2}}
\end{gathered}
$$

while at the interface $z=S_{0}(t)$, the first-order conditions are

$$
\begin{gathered}
S_{1} \frac{\partial T_{s 0}}{\partial z}+T_{s 1}=S_{1} \frac{\partial T_{l 0}}{\partial z}+T_{l 1} \\
S_{1} \frac{\partial c_{s 0}}{\partial z}+c_{s 1}=-m_{s}\left(S_{1} \frac{\partial T_{s 0}}{\partial z}+T_{s 1}\right) \\
S_{1} \frac{\partial c_{l 0}}{\partial z}+c_{l 1}=-m_{l}\left(S_{1} \frac{\partial T_{l 0}}{\partial z}+T_{l 1}\right) \\
\frac{\rho}{\mathrm{LeSt}} \frac{\mathrm{d} S_{1}}{\mathrm{~d} t}=k\left(S_{1} \frac{\partial^{2} T_{s 0}}{\partial z^{2}}+\frac{\partial T_{s 1}}{\partial z}\right)-\left(S_{1} \frac{\partial^{2} T_{l 0}}{\partial z^{2}}+\frac{\partial T_{l 1}}{\partial z}\right) \\
(1-\alpha)\left[\left(S_{1} \frac{\partial c_{l 0}}{\partial z}+c_{l 1}\right) \frac{\mathrm{d} S_{0}}{\mathrm{~d} t}+c_{l 0} \frac{\mathrm{d} S_{1}}{\mathrm{~d} t}\right]=D\left(S_{1} \frac{\partial^{2} c_{s 0}}{\partial z^{2}}+\frac{\partial c_{s 1}}{\partial z}\right)-\left(S_{1} \frac{\partial^{2} c_{l 0}}{\partial z^{2}}+\frac{\partial c_{l 1}}{\partial z}\right) .
\end{gathered}
$$

The first order perturbation terms are subject to boundary conditions which depend on the domain considered. For the infinite domain problem we have

$$
\begin{array}{lll}
T_{s 1}=0, & c_{s 1}=0 & \text { at } z \rightarrow-\infty, \\
T_{l 1}=0, & c_{l 1}=0 & \text { at } z \rightarrow+\infty,
\end{array}
$$

while for the semi-infinite problem we have

$$
\begin{aligned}
& T_{s 1}=0, \quad \frac{\partial c_{s 1}}{\partial z}=0 \quad \text { at } z=0, \\
& T_{l 1}=0, \quad c_{l 1}=0 \quad \text { at } z \rightarrow+\infty .
\end{aligned}
$$

We again make use of the similarity variable $\eta$, where the interface $z=S_{0}(t)$ is at $\eta=\lambda$. Changing variables from $z$ to $\eta$ in the governing PDEs (14a)-(14b), we obtain

$$
\begin{aligned}
\frac{\partial c_{s 1}}{\partial t} & =\frac{D}{4 t} \frac{\partial^{2} c_{s 1}}{\partial \eta^{2}}+\frac{\eta}{2 t} \frac{\partial c_{s 1}}{\partial \eta} \\
\frac{\partial T_{s 1}}{\partial t} & =\frac{\kappa \operatorname{Le}}{4 t} \frac{\partial^{2} T_{s 1}}{\partial \eta^{2}}+\frac{\eta}{2 t} \frac{\partial T_{s 1}}{\partial \eta} \\
\frac{\partial c_{l 1}}{\partial t} & =\frac{1}{4 t} \frac{\partial^{2} c_{l 1}}{\partial \eta^{2}}+\frac{\eta}{2 t} \frac{\partial c_{l 1}}{\partial \eta} \\
\frac{\partial T_{l 1}}{\partial t} & =\frac{\mathrm{Le}}{4 t} \frac{\partial^{2} T_{l 1}}{\partial \eta^{2}}+\frac{\eta}{2 t} \frac{\partial T_{l 1}}{\partial \eta}
\end{aligned}
$$


Seeking solutions to (17) by separation of variables and requiring the interface conditions (14c)$(14 \mathrm{~g})$ to have balanced powers of $t$, we conclude that these must take the form

$$
\begin{aligned}
c_{s 1} & =C_{c_{s}} t^{\beta} f_{c_{s}}(\eta), \\
T_{s 1} & =C_{T_{s}} t^{\beta} f_{T_{s}}(\eta), \\
c_{l 1} & =C_{c_{l}} t^{\beta} f_{c_{l}}(\eta), \\
T_{l 1} & =C_{T_{l}} t^{\beta} f_{T_{l}}(\eta), \\
S_{1} & =C_{S} t^{\beta+\frac{1}{2}},
\end{aligned}
$$

where $C_{j}$ are constants to be determined and $f_{j}$ are the corresponding functions in $\eta$ which we still have to find. We have chosen not to include $C_{j}$ into $f_{j}$ as this will be convenient later in the analysis. Observe that the stability of the problem is dictated by the position of the interface, $S_{1}$, since this has the highest power of $t$ and therefore it can be viewed as the least stable term. Hence, as the time exponent in the interface perturbation is $\beta+\frac{1}{2}$, we conclude that the system is unstable if $\beta>-\frac{1}{2}$.

Using (18), the interface conditions become

$$
\begin{gathered}
C_{S} \frac{T_{s 0}^{\prime}(\lambda)}{2}+C_{T_{s}} f_{T_{s}}(\lambda)=C_{S} \frac{T_{l 0}^{\prime}(\lambda)}{2}+C_{T_{l}} f_{T_{l}}(\lambda), \\
C_{S} \frac{c_{s 0}^{\prime}(\lambda)}{2}+C_{c_{s}} f_{c_{s}}(\lambda)=-m_{s}\left(C_{S} \frac{T_{s 0}^{\prime}(\lambda)}{2}+C_{T_{s}} f_{T_{s}}(\lambda)\right), \\
C_{S} \frac{c_{l 0}^{\prime}(\lambda)}{2}+C_{c_{l}} f_{c_{l}}(\lambda)=-m_{l}\left(C_{S} \frac{T_{l 0}^{\prime}(\lambda)}{2}+C_{T_{l}} f_{T_{l}}(\lambda)\right), \\
C_{S}\left(\beta+\frac{1}{2}\right) \frac{\rho}{\mathrm{LeSt}}=k\left(C_{S} \frac{T_{s 0}^{\prime \prime}(\lambda)}{4}+C_{T_{s}} \frac{f_{T_{s}}^{\prime}(\lambda)}{2}\right)-\left(C_{S} \frac{T_{l 0}^{\prime \prime}(\lambda)}{4}+C_{T_{l}} \frac{f_{T_{l}}^{\prime}(\lambda)}{2}\right), \\
(1-\alpha)\left[\left(C_{S} \frac{c_{l 0}^{\prime}(\lambda)}{2}+C_{c_{l}} f_{c_{l}}(\lambda)\right) \lambda+C_{S}\left(\beta+\frac{1}{2}\right) c_{i}\right] \\
=D\left(C_{S} \frac{c_{s 0}^{\prime \prime}(\lambda)}{4}+C_{c_{s}} \frac{f_{c_{s}}^{\prime}(\lambda)}{2}\right)-\left(C_{S} \frac{c_{l 0}^{\prime \prime}(\lambda)}{4}+C_{c_{l}} \frac{f_{c_{l}}^{\prime}(\lambda)}{2}\right),
\end{gathered}
$$

where prime denotes differentiation with respect to the similarity variable, $\eta$. Observe that we have a homogeneous linear system of five equations for the five unknowns $C_{c_{s}}, C_{c_{l}}, C_{T_{s}}, C_{T_{l}}$, and $C_{S}$. Thus, if the system is of full rank, the only solution is the trivial solution. In order to have non-trivial solutions we need the determinant of the system to be zero. This condition is the one that determines the value (or values) of $\beta$ where non-trivial solutions exist.

In order to find the functions $f_{j}(\eta)$ defined in (18), we need to solve the ODE obtained from using separation of variables in (17)

$$
\frac{\mu_{j}}{4} f_{j}^{\prime \prime}(\eta)+\frac{\eta}{2} f_{j}^{\prime}(\eta)-\beta f_{j}(\eta)=0
$$

where $\mu_{j}$ are known constants which are different for each field $j$ (for example, $\mu_{T_{s}}=\kappa \mathrm{Le}$ ). Introducing the change of variable $\zeta_{j}=\frac{\eta}{\sqrt{\mu_{j}}}$, the solution is given by $f\left(\zeta_{j}\right)=C_{j}^{(P)} P_{\beta}\left(\zeta_{j}\right)+$ 
$C_{j}^{(Q)} Q_{\beta}\left(\zeta_{j}\right)$, where we define

$$
P_{\beta}\left(\zeta_{j}\right)=e^{-\zeta_{j}^{2}} \zeta_{j} M\left(\beta+1, \frac{3}{2}, \zeta_{j}^{2}\right), \quad Q_{\beta}\left(\zeta_{j}\right)=e^{-\zeta_{j}^{2}} \zeta_{j} U\left(\beta+1, \frac{3}{2}, \zeta_{j}^{2}\right)
$$

Here $M\left(a, b, \zeta_{j}\right)$ and $U\left(a, b, \zeta_{j}\right)$ are Kummer's hypergeometric functions (see Abramowitz and Stegun (1964) for details). We will restrict all the subsequent analysis to the case where $\beta$ is real. Complex values of $\beta$ may occur but we have not considered these. Note that in the case where $\beta$ is a negative integer, the two Kummer's functions are no longer independent so we need to find a second independent solution. However, this case is not relevant for our analysis so we exclude it.

\section{Stability analysis of the semi-infinite domain problem}

In this section we perform the stability analysis for the semi-infinite domain problem. As a reminder, in the semi-infinite domain (7a) gives $c_{s 0}=\alpha c_{i}$ is constant, therefore $c_{s 0}^{\prime}(\eta)=$ $c_{s 0}^{\prime \prime}(\eta)=0$.

Boundary conditions for the semi-infinite domain problem are given by (16) so, using (18), we want $f_{T_{s}}(0)=0, f_{c_{s}}^{\prime}(0)=0$, and $f_{j}(\eta) \rightarrow 0$ as $\eta \rightarrow+\infty$ for the $j=T_{l}, c_{l}$. Yet, for $f_{T_{s}}(0)=0$ we have $f_{T_{s}}(0)=C_{T_{s}}^{(Q)} \sqrt{\pi} / \Gamma(1+\beta)=0$, and because we know that $\beta$ is not a negative integer, we require $C_{T_{s}}^{(Q)}=0$. For $f_{c_{s}}^{\prime}(0)=0$ we therefore have

$$
f_{c_{s}}^{\prime}(0)=\frac{C_{c_{s}}^{(P)}}{\sqrt{D}}+\frac{C_{c_{s}}^{(Q)}}{\sqrt{D}} U\left(\beta+1, \frac{3}{2}, 0\right)=0
$$

and we note that because $\beta$ is not a negative integer $U(a, b, z)$ blows up at zero. Therefore, we need $C_{c_{s}}^{(Q)}=0$ which implies $C_{c_{s}}^{(P)}=0$. Then, the perturbation has the form $f_{c_{s}}(\eta)=0$. For the liquid phase we need $f_{j}(\eta)$ with $j=T_{l}, c_{l}$ to decay as $\eta \rightarrow+\infty$. From Abramowitz and Stegun (1964), we know that

$$
\begin{aligned}
& P_{\beta}(\eta)=\eta^{2 \beta} \frac{\sqrt{\pi}}{2 \Gamma(\beta+1)}\left(\sum_{n=0}^{N-1} \frac{\left(\frac{1}{2}-\beta\right)_{n}(-\beta)_{n}}{n !} \eta^{-2 n}+\mathcal{O}\left(\eta^{-2 N}\right)\right) \\
& Q_{\beta}(\eta)=e^{-\eta^{2}} \eta^{-2 \beta-1}\left(\sum_{n=0}^{N-1}(-1)^{n} \frac{(\beta+1)_{n}\left(\beta+\frac{1}{2}\right)_{n}}{n !} \eta^{-2 n}+\mathcal{O}\left(\eta^{-2 N}\right)\right),
\end{aligned}
$$

as $\eta \rightarrow+\infty$, where $(a)_{n}=\Gamma(a+n) / \Gamma(a)$ is Pochhammer's symbol. Notice that $Q_{\beta}(\eta)$ decays for any choice of $\beta$. In order for $P_{\beta}(\eta)$ to satisfy the boundary conditions, we need $\beta<0$. Therefore, if we want $f_{j}(\eta)$ with $j=T_{l}, c_{l}$ to satisfy the boundary conditions at $\eta \rightarrow+\infty$ we require either $\beta<0$ or $C_{j}^{(P)}=0$. Therefore, we must study separately the cases $\beta \geqslant 0$ and $\beta<0$.

Summarising, for the semi-infinite domain, we can conclude that the perturbations for $\beta \geqslant 0$ 
are

$$
\begin{aligned}
& c_{s 1}(t, \eta)=0, \\
& T_{s 1}(t, \eta)=C_{T_{s}} t^{\beta} P_{\beta}\left(\frac{\eta}{\sqrt{\kappa \mathrm{Le}}}\right), \\
& c_{l 1}(t, \eta)=C_{c_{l}} t^{\beta} Q_{\beta}(\eta), \\
& T_{l 1}(t, \eta)=C_{T_{l}} t^{\beta} Q_{\beta}\left(\frac{\eta}{\sqrt{\mathrm{Le}}}\right),
\end{aligned}
$$

while for $\beta<0$ we have

$$
\begin{aligned}
& c_{s 1}(t, \eta)=0 \\
& T_{s 1}(t, \eta)=C_{T_{s}} t^{\beta} P_{\beta}\left(\frac{\eta}{\sqrt{\kappa \mathrm{Le}}}\right) \\
& c_{l 1}(t, \eta)=t^{\beta}\left(C_{c_{l}}^{(P)} P_{\beta}(\eta)+C_{c_{l}}^{(Q)} Q_{\beta}(\eta)\right), \\
& T_{l 1}(t, \eta)=t^{\beta}\left(C_{T_{l}}^{(P)} P_{\beta}\left(\frac{\eta}{\sqrt{\mathrm{Le}}}\right)+C_{T_{l}}^{(Q)} Q_{\beta}\left(\frac{\eta}{\sqrt{\mathrm{Le}}}\right)\right),
\end{aligned}
$$

where the $C_{j}$ 's are constants.

\subsection{No instability for $\beta \geqslant 0$}

In the case $\beta \geqslant 0$ we have (20), therefore we do not need to determine $C_{c_{s}}$ as $f_{c_{s}}(\eta)=0$. Now, the system (19) reduces to an overdetermined system of five equations with four unknowns. In order to have non-trivial solutions we require all $4 \times 4$ minors to be equal to zero. This will give five conditions, which can be shown to be equivalent to two algebraic conditions. Using the relations

and defining

$$
\frac{T_{s 0}^{\prime \prime}(\eta)}{T_{s 0}^{\prime}(\eta)}=-\frac{2 \eta}{\kappa \mathrm{Le}}, \quad \frac{T_{l 0}^{\prime \prime}(\eta)}{T_{l 0}^{\prime}(\eta)}=-\frac{2 \eta}{\mathrm{Le}}, \quad \text { and } \quad \frac{c_{l 0}^{\prime \prime}(\eta)}{c_{l 0}^{\prime}(\eta)}=-2 \eta
$$

$$
\begin{aligned}
& \mathcal{M}_{\beta}(\lambda ; \mu)=\lim _{\eta \rightarrow \lambda} \frac{d}{d \eta} \ln \left(P_{\beta}\left(\frac{\eta}{\sqrt{\mu}}\right)\right), \\
& \mathcal{U}_{\beta}(\lambda ; \mu)=\lim _{\eta \rightarrow \lambda} \frac{d}{d \eta} \ln \left(Q_{\beta}\left(\frac{\eta}{\sqrt{\mu}}\right)\right),
\end{aligned}
$$

we can rewrite the two algebraic conditions as

$$
\begin{aligned}
k\left(\mathcal{M}_{\beta}(\lambda ; \kappa \mathrm{Le})+\right. & \left.\frac{2 \lambda}{\kappa \mathrm{Le}}\right) T_{s 0}^{\prime}(\lambda)-\left(\mathcal{U}_{\beta}(\lambda ; \mathrm{Le})+\frac{2 \lambda}{\mathrm{Le}}\right) T_{l 0}^{\prime}(\lambda)+4\left(\beta+\frac{1}{2}\right) \frac{\rho}{\mathrm{LeSt}}=0, \\
& -\left(\mathcal{U}_{\beta}(\lambda ; 1)+2 \lambda\right) c_{l 0}^{\prime}(\lambda)+4\left(\beta+\frac{1}{2}\right)(1-\alpha) c_{i}=0 .
\end{aligned}
$$

We want to show that for any parameter choice there is no value of $\beta$ that solves the system (22)-(23). Using the definition of $c_{i}$ we know $\left[c_{i}-1\right] / F(\lambda)=(1-\alpha) c_{i}$, hence we can rewrite (23) as

$$
\left(1-2 \lambda^{2}(\beta+1) \frac{U\left(\beta+2, \frac{5}{2}, \lambda^{2}\right)}{U\left(\beta+1, \frac{3}{2}, \lambda^{2}\right)}\right)+2\left(\beta+\frac{1}{2}\right)=0 .
$$


We do not give details here but it can be shown analytically that if $\beta \geqslant 0$ there are no finite values of $\lambda$.

We can conclude that, for the semi-infinite domain and $\beta \geqslant 0$ there are no perturbations that satisfy the stability problem. Therefore the temperature and concentration fields are always stable, so the instability of the problem will be driven by the position of the interface. This is reasonable, in light of the fact that the individual diffusion PDEs are stable. This motivates us to consider the situation where only the interface develops an instability, i.e. $-\frac{1}{2} \leqslant \beta<0$.

\subsection{Instability for $-\frac{1}{2} \leqslant \beta<0$}

For this case, the interface can go unstable even though the concentration and temperature fields are stable. From (18), this means $-\frac{1}{2} \leqslant \beta<0$. For $\beta<0$ (and $\beta$ not a negative integer) we have (21). Therefore we have 6 unknowns and 5 equations to determine them. Normalising the perturbations with their value at the interface $(\eta=\lambda)$ and after some manipulation, the system (19) can be written as $A_{\text {semi }}(\beta) \mathbf{C}_{\text {semi }}(\beta)=\mathbf{0}$ where

$$
A_{\text {semi }}(\beta)=\left(\begin{array}{llllll}
\mathbf{A}_{\text {semi }}^{1}(\beta) & \mathbf{A}_{\text {semi }}^{2}(\beta) & \mathbf{A}_{\text {semi }}^{3}(\beta) & \mathbf{A}_{\text {semi }}^{4}(\beta) & \mathbf{A}_{\text {semi }}^{5}(\beta) & \mathbf{A}_{\text {semi }}^{6}(\beta)
\end{array}\right),
$$

with

$$
\begin{aligned}
& \mathbf{A}_{\text {semi }}^{1}(\beta)=\left(\begin{array}{c}
0 \\
1 \\
0 \\
\frac{k}{2} \mathcal{M}_{\beta}(\lambda ; \kappa \mathrm{Le}) \\
0
\end{array}\right), \quad \mathbf{A}_{\text {semi }}^{2}(\beta)=\left(\begin{array}{c}
1 \\
0 \\
0 \\
-\frac{1}{2} \mathcal{M}_{\beta}(\lambda ; \mathrm{Le}) \\
0
\end{array}\right) \\
& \mathbf{A}_{\text {semi }}^{3}(\beta)=\left(\begin{array}{c}
1 \\
0 \\
0 \\
-\frac{1}{2} \mathcal{U}_{\beta}(\lambda ; \mathrm{Le}) \\
0
\end{array}\right), \quad \mathbf{A}_{\text {semi }}^{4}(\beta)=\left(\begin{array}{c}
0 \\
0 \\
1 \\
0 \\
-\frac{1}{2} \mathcal{M}_{\beta}(\lambda ; 1)
\end{array}\right) \text {, } \\
& \mathbf{A}_{\text {semi }}^{5}(\beta)=\left(\begin{array}{c}
0 \\
0 \\
1 \\
0 \\
-\frac{1}{2} \mathcal{U}_{\beta}(\lambda ; 1)
\end{array}\right), \quad \mathbf{A}_{s e m i}^{6}(\beta)=\left(\begin{array}{c}
\frac{1}{2} T_{l 0}^{\prime}(\lambda) \\
\frac{1}{2} T_{s 0}^{\prime}(\lambda) \\
\frac{1}{2} c_{l 0}^{\prime}(\lambda) \\
\frac{1}{4}\left[k T_{s 0}^{\prime \prime}(\lambda)-T_{l 0}^{\prime \prime}(\lambda)\right]-\left(\beta+\frac{1}{2}\right) \frac{\rho}{\operatorname{LeSt}} \\
-\frac{1}{4} c_{l 0}^{\prime \prime}(\lambda)-\left(\beta+\frac{1}{2}\right)(1-\alpha) c_{i}
\end{array}\right)
\end{aligned}
$$

and

$$
\mathbf{C}_{s e m i}(\beta)=\left(C_{T_{s}}(\beta), C_{T_{l}}^{(P)}(\beta), C_{T_{l}}^{(Q)}(\beta), C_{c_{l}}^{(P)}(\beta), C_{c_{l}}^{(Q)}(\beta), C_{S}(\beta)\right)^{T} .
$$

The components in $\mathbf{C}_{\text {semi }}(\beta)$ are the relative sizes of the perturbations.

If we take the $5 \times 5$ minor given by the first five columns we find that the determinant is

$$
\operatorname{det}\left(A_{\text {semi }}(\beta)\right)=\frac{1}{4}\left(\mathcal{M}_{\beta}(\lambda ; \text { Le })-\mathcal{U}_{\beta}(\lambda ; \text { Le })\right)\left(\mathcal{M}_{\beta}(\lambda ; 1)-\mathcal{U}_{\beta}(\lambda ; 1)\right) .
$$

We can show analytically that if $\beta>-1$ then

$$
\mathcal{M}_{\beta}(\lambda ; \mu)-\mathcal{U}_{\beta}(\lambda ; \mu) \neq 0
$$




\begin{tabular}{|c||c|c|c|c|}
\hline$\beta$ & 0 & $-\frac{1}{4}$ & $-\frac{1}{2}$ & $-\frac{3}{4}$ \\
\hline \hline$C_{T_{s}}$ & $-4.0043 \cdot 10^{-5}$ & $-4.0045 \cdot 10^{-5}$ & $-4.0047 \cdot 10^{-5}$ & $-4.0047 \cdot 10^{-5}$ \\
$C_{T_{l}}^{(P)}$ & $-1.0801 \cdot 10^{-4}$ & $-1.0119 \cdot 10^{-4}$ & $-9.3493 \cdot 10^{-5}$ & $-8.4531 \cdot 10^{-5}$ \\
$C_{T_{l}}^{(Q)}$ & $7.5287 \cdot 10^{-5}$ & $6.8463 \cdot 10^{-5}$ & $6.0764 \cdot 10^{-5}$ & $5.1801 \cdot 10^{-5}$ \\
$C_{c_{l}}^{(P)}$ & $-1.0059 \cdot 10^{-4}$ & $-3.8257 \cdot 10^{-5}$ & 0 & $1.3124 \cdot 10^{-5}$ \\
$C_{c_{l}}^{(Q)}$ & $9.9999 \cdot 10^{-1}$ & $9.9999 \cdot 10^{-1}$ & $9.9999 \cdot 10^{-1}$ & $9.9999 \cdot 10^{-1}$ \\
$C_{S}$ & $1.0939 \cdot 10^{-3}$ & $1.0939 \cdot 10^{-3}$ & $1.0940 \cdot 10^{-3}$ & $1.0940 \cdot 10^{-3}$ \\
\hline
\end{tabular}

Table 2: Amplitudes of the perturbations $\mathbf{u}_{\text {semi }}(\beta)$ for different values of $\beta$. The values shown correspond to normalised vectors.

for any $\lambda \neq 0$ and $\mu>0$. Thus, the matrix is always fully ranked and from the rank-nullity theorem we conclude that the kernel of the matrix will be of size one.

The amplitudes $\mathbf{C}_{\text {semi }}(\beta)$ are given by linear combinations of the elements of the kernel. For the semi-infinite domain the kernel is generated by the vector

$$
\mathbf{u}_{s e m i}(\beta)=\left(\begin{array}{c}
-\frac{1}{2} T_{s 0}^{\prime}(\lambda) \\
-\frac{k\left(\mathcal{M}_{\beta}(\lambda ; \kappa \mathrm{Le})+\frac{2 \lambda}{\kappa \mathrm{Le}}\right) T_{s 0}^{\prime}(\lambda)-\left(\mathcal{U}_{\beta}(\lambda ; \mathrm{Le})+\frac{2 \lambda}{\mathrm{Le}}\right) T_{l 0}^{\prime}(\lambda)+4\left(\beta+\frac{1}{2}\right) \frac{\rho}{\mathrm{LeSt}}}{2\left[\mathcal{M}_{\beta}(\lambda ; \mathrm{Le})-\mathcal{U}_{\beta}(\lambda ; \mathrm{Le})\right]} \\
\frac{k\left(\mathcal{M}_{\beta}(\lambda ; \kappa \mathrm{Le})+\frac{2 \lambda}{\kappa \mathrm{Le}}\right) T_{s 0}^{\prime}(\lambda)-\left(\mathcal{M}_{\beta}(\lambda ; \mathrm{Le})+\frac{2 \lambda}{\mathrm{Le}}\right) T_{l 0}^{\prime}(\lambda)+4\left(\beta+\frac{1}{2}\right) \frac{\rho}{\mathrm{LeSt}}}{2\left[\mathcal{M}_{\beta}(\lambda ; \mathrm{Le})-\mathcal{U}_{\beta}(\lambda ; \mathrm{Le})\right]} \\
-\frac{-\left(\mathcal{U}_{\beta}(\lambda ; 1)+2 \lambda\right) c_{l 0}^{\prime}(\lambda)+4\left(\beta+\frac{1}{2}\right)(1-\alpha) c_{i}}{2\left[\mathcal{M}_{\beta}(\lambda ; 1)-\mathcal{U}_{\beta}(\lambda ; 1)\right]} \\
\frac{-\left(\mathcal{M}_{\beta}(\lambda ; 1)+2 \lambda\right) c_{l 0}^{\prime}(\lambda)+4\left(\beta+\frac{1}{2}\right)(1-\alpha) c_{i}}{2\left[\mathcal{M}_{\beta}(\lambda ; 1)-\mathcal{U}_{\beta}(\lambda ; 1)\right]} \\
1
\end{array}\right) .
$$

As we have mentioned before, we can show analytically that the denominators of the elements of the kernel do not vanish for $\beta>-1, \lambda \neq 0$ and $\mu>0$ so the vector is well defined.

Therefore we can always find unstable perturbations of the system. The most unstable mode is the one given by $\beta \rightarrow 0$ (but note that the case $\beta=0$ is out of the scope of the analysis as the boundary conditions are not satisfied). Then the perturbation of the interface can be written as

$$
S(t)=2 \lambda t^{\frac{1}{2}}+\delta t^{\frac{1}{2}-\epsilon}
$$

for any $\epsilon>0$, so the growth rate of the most unstable perturbation tends to a multiple of the base state. When $\beta \rightarrow 0$, if $\lambda>0$ and $\mu>0$, we have

$$
\mathcal{M}_{\beta}(\lambda ; \mu)=\frac{2 e^{-\frac{\lambda^{2}}{\mu}}}{\sqrt{\pi \mu} \operatorname{erf}\left(\frac{\lambda}{\sqrt{\mu}}\right)}, \quad \text { and } \quad \mathcal{U}_{\beta}(\lambda ; \mu)=-\frac{2 e^{-\frac{\lambda^{2}}{\mu}}}{\sqrt{\pi \mu} \operatorname{erfc}\left(\frac{\lambda}{\sqrt{\mu}}\right)}
$$

so the vector of the kernel can be simplified.

In Figure 4 we show the shape of the perturbations for the semi-infinite domain problem for different values of $\beta$. The parameter values are the ones to model solidification of metallurgical 


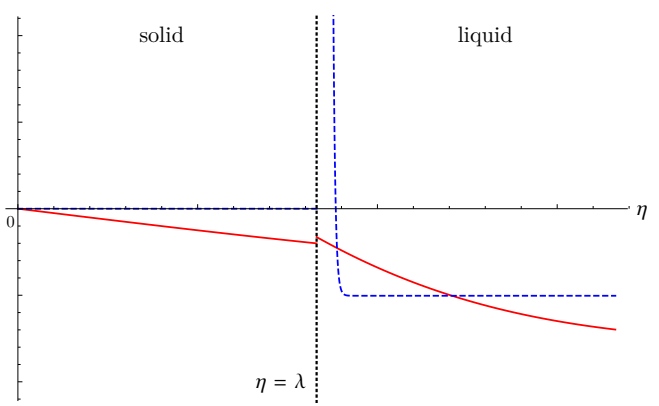

(a) $\beta=0$

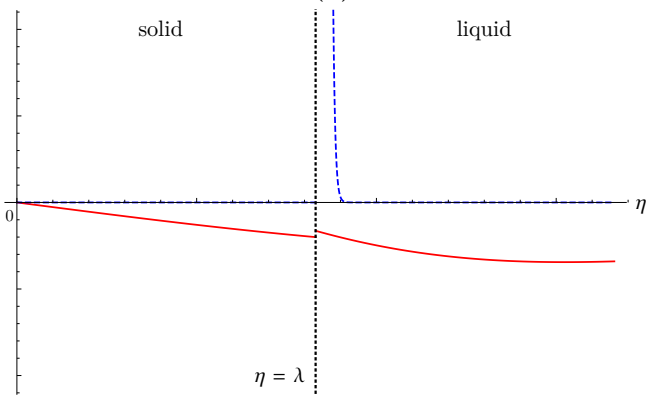

(c) $\beta=-\frac{1}{2}$

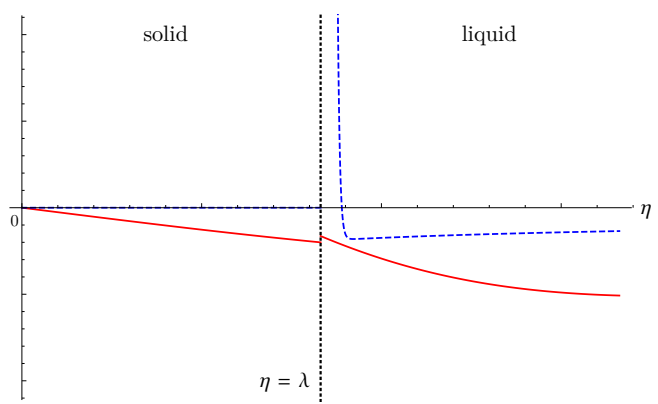

(b) $\beta=-\frac{1}{4}$

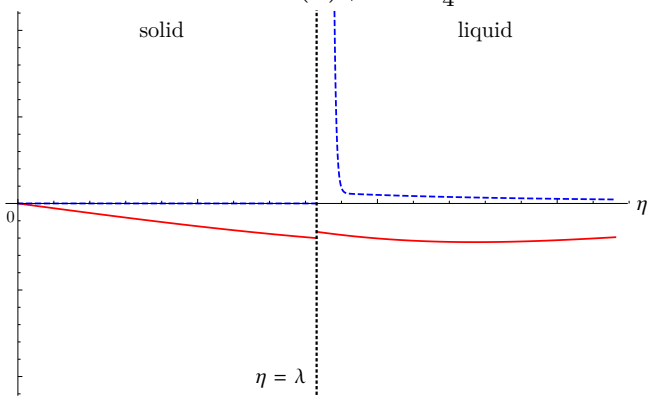

(d) $\beta=-\frac{3}{4}$

FIG. 4: Perturbations for the semi-infinite domain for different values of $\beta$ using the amplitudes given by $\mathbf{u}_{\text {semi }}(\beta)$ (numerical values shown in Table 2). The domain of the perturbations is $\eta \geqslant 0$ as we are in the semi-infinite domain problem defined by $z \geqslant 0$ and $t>0$. The scaling is the same in all the plots so they are directly comparable. The parameters of the system have been set to $\mathrm{St}=0.77, \mathrm{Le}=134, \rho=0.95, c_{p}=1, k=2.91, \kappa=3.06, m_{s}=0.69, m_{l}=346$, $D=1.76 \cdot 10^{-2}, \alpha=2 \cdot 10^{-3}$ and $T_{l \infty}=0$. 
grade silicon in a copper mould (see Table 1 ). We observe that when $\beta=0$ boundary conditions at infinity are not satisfied and the solution is equivalent to the base state, so it only modifies the amplitudes of the base state solutions, keeping their shape. The case $\beta=-\frac{1}{4}$ is in the unstable regime as $-\frac{1}{2}<\beta<0$. We observe that there is a spike in $c_{l 1}$ just ahead of the interface due to rejection of impurities during solidification. However outside this narrow spike it has a negative contribution to the liquid concentration. The contribution of the temperature perturbations is negative in both phases. The case $\beta=-\frac{1}{2}$ corresponds to the marginally stable perturbation. Observe that in this case the temperature perturbations are still negative, while the perturbation in the liquid concentration still has a spike just ahead of the interface which again quickly decays to zero. This can be shown analytically as the fourth component of $\mathbf{u}_{\text {inf }}$ is identically zero if $\beta=-\frac{1}{2}$ (the spike is due to the fifth component). Finally, the case $\beta=-\frac{3}{4}$ corresponds to a stable perturbation. In that case, we observe that the perturbation of the liquid concentration is positive.

The evolution of the perturbations for different values of $\beta$ shown in the plots hints that the perturbation of the concentration in the liquid is the one dominating the stability of the problem. If we look at the amplitudes of the perturbations shown in Table 2 we observe that the largest amplitude is $C_{c_{l}}^{(Q)}$ for any choice of $\beta$, agreeing with the idea that the dominating perturbation is $c_{l 1}$. We observe as well that $C_{S}$ is much smaller than $C_{c_{l}}^{(Q)}$ but much larger than any other amplitude. Therefore, one could expect that $c_{l 1}$ and $S_{1}$ to determine the behaviour of the system, with much less effect of the other perturbations. Finally, notice that the small variations in the amplitudes $C_{T_{s}}$ and $C_{S}$ are due to the normalization, as from the form of $\mathbf{u}_{\text {semi }}(\beta)$ we observe that they do not depend on $\beta$.

\section{Stability analysis of the infinite domain problem}

Having considered a detailed stability analysis for the solidification front in the semi-infinite case, we now turn our attention to the corresponding analysis for the infinite domain. We should remark that the infinite domain problem presented in this section is the same geometry as in Coriell et al. (1999). However, in their stability analysis, the perturbations introduced

are of the particular form $e^{-\frac{\eta^{2}}{4}} \mathcal{D}_{-2 \beta-1}(\eta)$, where $\mathcal{D}_{\nu}(\eta)$ is a parabolic cylinder function. This is a particular case of $Q_{\beta}(\eta)$ as for certain parameter choices it reduces to $e^{\frac{-\eta^{2}}{4}} \mathcal{D}_{-2 \beta-1}(\eta)$. Therefore, the approach in Coriell et al. (1999) does not consider the contribution of $P_{\beta}(\eta)$, which we will show to be necessary for understanding instabilities that may develop in the solidification boundary. In addition, Coriell et al. (1999) does not discuss the fact that, even though the velocity of the interface might decay in time, if this decay is not strong enough the interface may still develop instabilities, as we show in our analysis.

The boundary conditions are given by (15) and translated into the self-similar coordinate system we have

$$
\begin{aligned}
& f_{j}(\eta) \rightarrow 0 \text { when } \quad \eta \rightarrow-\infty \text { for } j=T_{s}, c_{s} \\
& f_{j}(\eta) \rightarrow 0 \text { when } \quad \eta \rightarrow+\infty \text { for } j=T_{l}, c_{l} .
\end{aligned}
$$

The analysis for $f_{T_{l}}$ and $f_{c_{l}}$ when $\eta \rightarrow-\infty$ is equivalent to the analysis for $f_{T_{s}}$ and $f_{c_{s}}$ when $\eta \rightarrow+\infty$ as they behave similarly in the limit. Therefore, the analysis done in the previous section for the liquid perturbations can be applied here. Observe that $Q_{\beta}\left(\zeta_{j}\right)$ is discontinuous at $\zeta_{j}=0$, which is in the solid phase. Therefore, because we want the perturbations to be 
continuous, in the solid phase we need $C_{j}^{(Q)}=0$ for $j=T_{s}, c_{s}$. We can conclude that the perturbations for $\beta \geqslant 0$ are

$$
\begin{aligned}
c_{s 1}(t, \eta) & =0, \\
T_{s 1}(t, \eta) & =0, \\
c_{l 1}(t, \eta) & =C_{c_{l}} t^{\beta} Q_{\beta}(\eta), \\
T_{l 1}(t, \eta) & =C_{T_{l}} t^{\beta} Q_{\beta}\left(\frac{\eta}{\sqrt{\mathrm{Le}}}\right),
\end{aligned}
$$

while if $\beta<0$ we have

$$
\begin{aligned}
& c_{s 1}(t, \eta)=C_{c_{s}} t^{\beta} P_{\beta}\left(\frac{\eta}{\sqrt{D}}\right), \\
& T_{s 1}(t, \eta)=C_{T_{s}} t^{\beta} P_{\beta}\left(\frac{\eta}{\sqrt{\kappa \mathrm{Le}}}\right) \\
& c_{l 1}(t, \eta)=t^{\beta}\left(C_{c_{l}}^{(P)} P_{\beta}(\eta)+C_{c_{l}}^{(Q)} Q_{\beta}(\eta)\right), \\
& T_{l 1}(t, \eta)=t^{\beta}\left(C_{T_{l}}^{(P)} P_{\beta}\left(\frac{\eta}{\sqrt{\mathrm{Le}}}\right)+C_{T_{l}}^{(Q)} Q_{\beta}\left(\frac{\eta}{\sqrt{\mathrm{Le}}}\right)\right),
\end{aligned}
$$

where $C_{j}$ are undetermined constants.

As was the case for the semi-infinite domain, we may show that there are no non-trivial perturbations for $\beta \geqslant 0$. Therefore, we restrict our attention to the case of $-\frac{1}{2} \leqslant \beta<0$.

\subsection{Instability for $-\frac{1}{2} \leqslant \beta<0$}

For this case, the interface can go unstable even though the concentration and temperature fields are stable. We use the perturbations listed in (32a) so now we have 7 unknowns and 5 equations. The system (19) can be written as $A_{\text {inf }}(\beta) \mathbf{C}_{\text {inf }}(\beta)=\mathbf{0}$ where

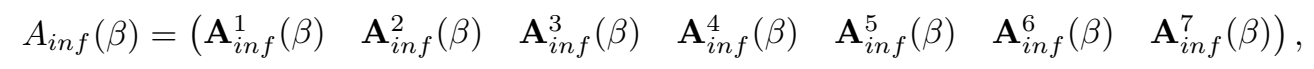

with

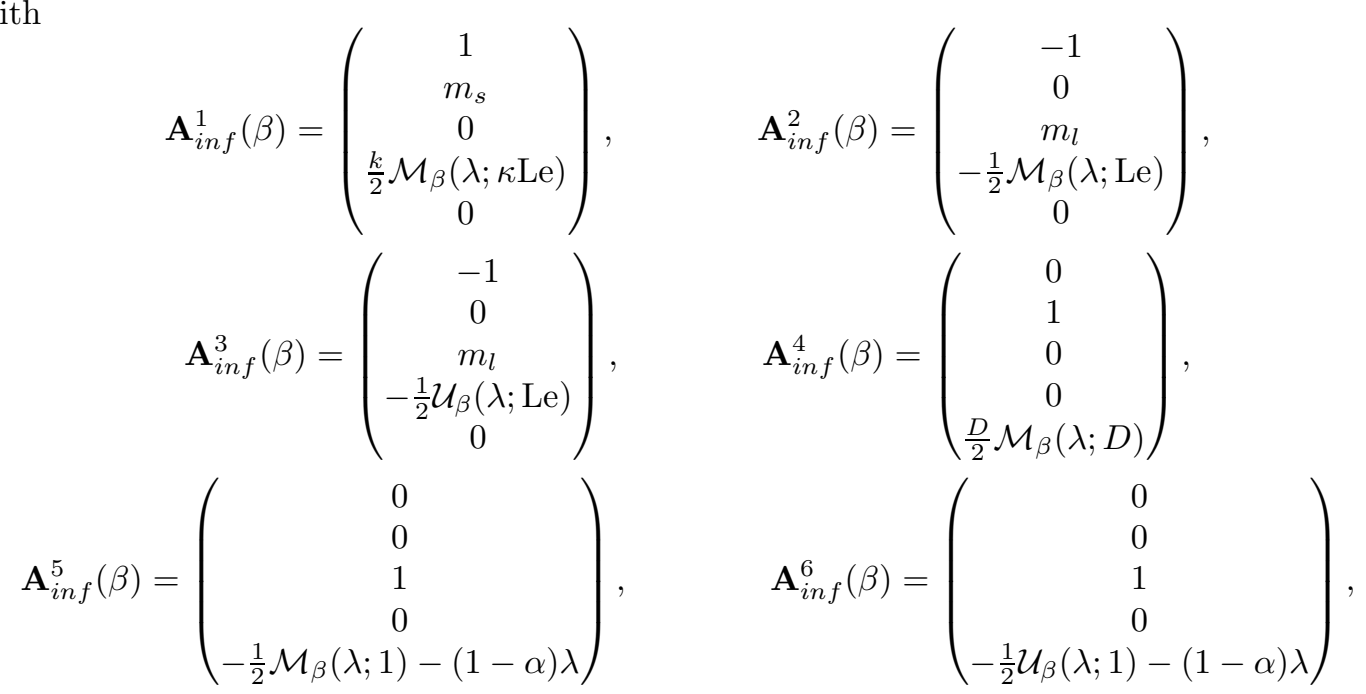

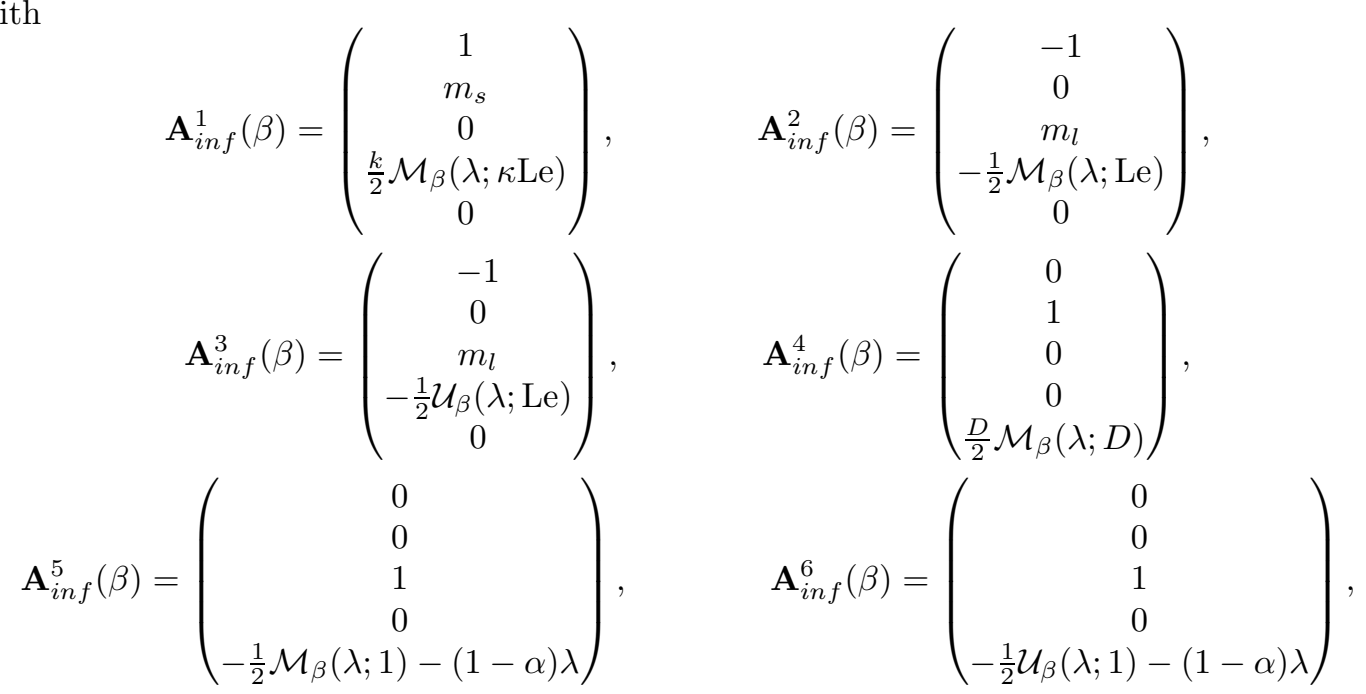

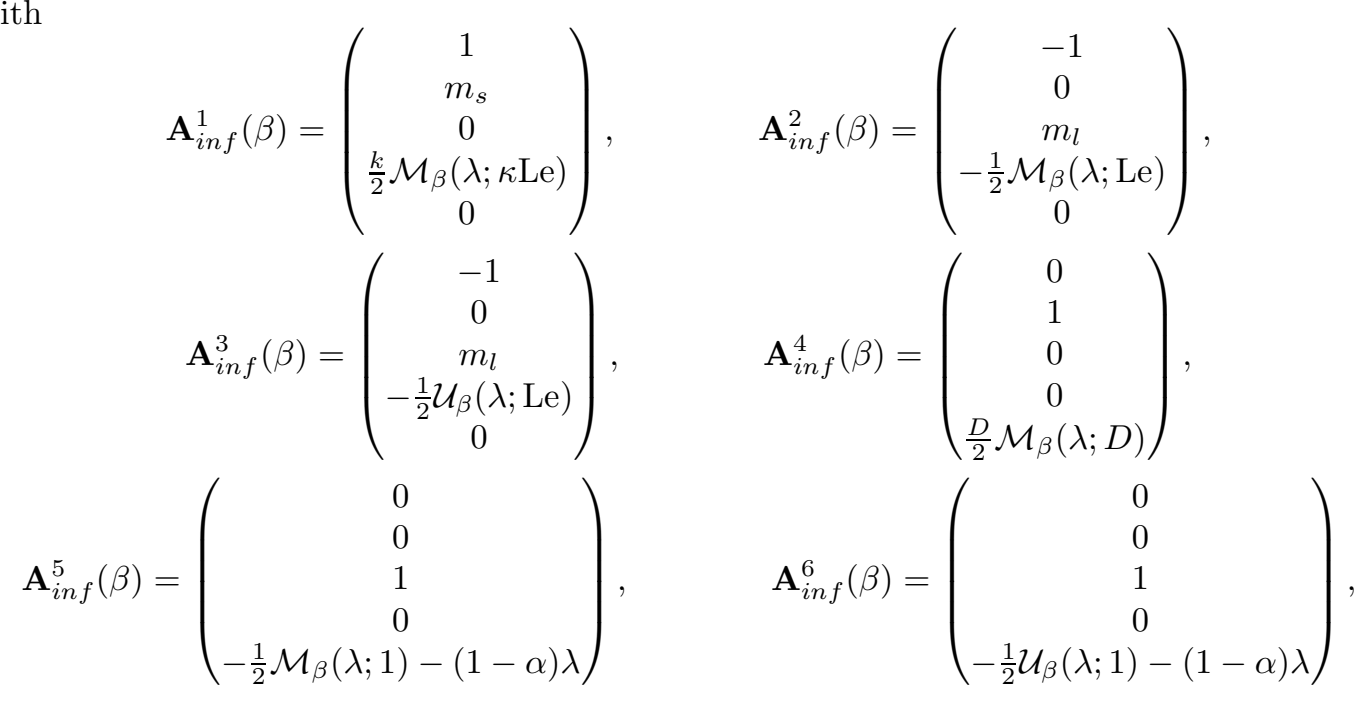


INSTABILITY IN THE SELF-SIMILAR MOTION OF A PLANAR SOLIDIFICATION FRONT 19 of 25

$$
\mathbf{A}_{i n f}^{7}(\beta)=\left(\begin{array}{c}
\frac{1}{2}\left[T_{s 0}^{\prime}(\lambda)-T_{l 0}^{\prime}(\lambda)\right] \\
\frac{1}{2}\left[m_{s} T_{s 0}^{\prime}(\lambda)+c_{s 0}^{\prime}(\lambda)\right] \\
\frac{1}{2}\left[m_{l} T_{l 0}^{\prime}(\lambda)+c_{l 0}^{\prime}(\lambda)\right] \\
\frac{1}{4}\left[k T_{s 0}^{\prime \prime}(\lambda)-T_{l 0}^{\prime \prime}(\lambda)\right]-\left(\beta+\frac{1}{2}\right) \frac{\rho}{\operatorname{LeSt}} \\
\frac{1}{4}\left[D c_{s 0}^{\prime \prime}(\lambda)-c_{l 0}^{\prime \prime}(\lambda)\right]-(1-\alpha)\left[\left(\beta+\frac{1}{2}\right) c_{i}+\lambda \frac{c_{l 0}^{\prime}(\lambda)}{2}\right]
\end{array}\right)
$$

and

$$
\mathbf{C}_{i n f}(\beta)=\left(C_{T_{s}}(\beta), C_{T_{l}}^{(P)}(\beta), C_{T_{l}}^{(Q)}(\beta), C_{c_{s}}(\beta), C_{c_{l}}^{(P)}(\beta), C_{c_{l}}^{(Q)}(\beta), C_{S}(\beta)\right)^{T}
$$

Taking the minor generated by columns $1,2,3,5$ and 6 of $A_{\text {inf }}(\beta)$ we can show, as in the semi-infinite domain, that if $\beta>-1$ the matrix is fully ranked. Again, by the rank-nullity theorem we can conclude that the size of the kernel will be exactly two. Thus, we can always find non-trivial values of $C_{i n f}$ by taking linear combinations of the elements of the kernel. The kernel for the infinite domain problem is generated by the vectors

$$
\mathbf{u}_{i n f}(\beta)=\left(\begin{array}{c}
-\frac{1}{2} T_{s 0}^{\prime}(\lambda) \\
-\frac{k\left(\mathcal{M}_{\beta}(\lambda ; \kappa \mathrm{Le})+\frac{2 \lambda}{\kappa \mathrm{Le}}\right) T_{s 0}^{\prime}(\lambda)-\left(\mathcal{U}_{\beta}(\lambda ; \mathrm{Le})+\frac{2 \lambda}{\mathrm{Le}}\right) T_{l 0}^{\prime}(\lambda)+4\left(\beta+\frac{1}{2}\right) \frac{\rho}{\mathrm{LeSt}}}{2\left[\mathcal{M}_{\beta}(\lambda ; \mathrm{Le})-\mathcal{U}_{\beta}(\lambda ; \mathrm{Le})\right]} \\
\frac{k\left(\mathcal{M}_{\beta}(\lambda ; \kappa \mathrm{Le})+\frac{2 \lambda}{\kappa \mathrm{Le}}\right) T_{s 0}^{\prime}(\lambda)-\left(\mathcal{M}_{\beta}(\lambda ; \mathrm{Le})+\frac{2 \lambda}{\mathrm{Le}}\right) T_{l 0}^{\prime}(\lambda)+4\left(\beta+\frac{1}{2}\right) \frac{\rho}{\mathrm{LeSt}}}{2\left[\mathcal{M}_{\beta}(\lambda ; \mathrm{Le})-\mathcal{U}_{\beta}(\lambda ; \mathrm{Le})\right]} \\
-\frac{-\left(\mathcal{U}_{\beta}(\lambda ; 1)+2 \lambda\right) c_{l 0}^{\prime}(\lambda)+4\left(\beta+\frac{1}{2}\right)(1-\alpha) c_{i}}{2\left[\mathcal{M}_{\beta}(\lambda ; 1)-\mathcal{U}_{\beta}(\lambda ; 1)\right]} \\
\frac{-\left(\mathcal{M}_{\beta}(\lambda ; 1)+2 \lambda\right) c_{l 0}^{\prime}(\lambda)+4\left(\beta+\frac{1}{2}\right)(1-\alpha) c_{i}}{2\left[\mathcal{M}_{\beta}(\lambda ; 1)-\mathcal{U}_{\beta}(\lambda ; 1)\right]} \\
+\frac{c_{s 0}^{\prime}(\lambda)}{m_{s}}\left(\begin{array}{c}
1 \\
\frac{k \mathcal{M}_{\beta}(\lambda ; \kappa \mathrm{Le})-\mathcal{U}_{\beta}(\lambda ; \mathrm{Le})}{2\left(\mathcal{M}_{\beta}(\lambda ; \mathrm{Le})-\mathcal{U}_{\beta}(\lambda ; \mathrm{Le})\right)} \\
\frac{k \mathcal{M}_{\beta}(\lambda ; \kappa \mathrm{Le})-\mathcal{M}_{\beta}(\lambda ; \mathrm{Le})}{2\left(\mathcal{M}_{\beta}(\lambda ; \mathrm{Le})-\mathcal{U}_{\beta}(\lambda ; \mathrm{Le})\right)} \\
0 \\
-\frac{m_{l}\left(\mathcal{U}_{\beta}(\lambda ; 1)+2 \lambda\right)}{2\left(\mathcal{M}_{\beta}(\lambda ; 1)-\mathcal{U}_{\beta}(\lambda ; 1)\right)} \\
\frac{m_{l}\left(\mathcal{M}_{\beta}(\lambda ; 1)+2 \lambda\right)}{2\left(\mathcal{M}_{\beta}(\lambda ; 1)-\mathcal{U}_{\beta}(\lambda ; 1)\right)} \\
0
\end{array}\right)
\end{array}\right.
$$




\begin{tabular}{|c||c|c|c|c|}
\hline$\beta$ & 0 & $-\frac{1}{4}$ & $-\frac{1}{2}$ & $-\frac{3}{4}$ \\
\hline \hline$C_{T_{s}}$ & $-8.7473 \cdot 10^{-5}$ & $-8.7519 \cdot 10^{-5}$ & $-8.7548 \cdot 10^{-5}$ & $-8.7559 \cdot 10^{-5}$ \\
$C_{T_{l}}^{(P)}$ & $-3.1391 \cdot 10^{-4}$ & $-2.7772 \cdot 10^{-4}$ & $-2.3646 \cdot 10^{-4}$ & $-1.8812 \cdot 10^{-4}$ \\
$C_{T_{l}}^{(Q)}$ & $2.6927 \cdot 10^{-4}$ & $2.3306 \cdot 10^{-4}$ & $1.9178 \cdot 10^{-4}$ & $1.4344 \cdot 10^{-4}$ \\
$C_{c_{s}}$ & 0 & 0 & 0 & 0 \\
$C_{C_{l}}^{(P)}$ & $-8.6577 \cdot 10^{-4}$ & $-3.3837 \cdot 10^{-4}$ & 0 & $1.2301 \cdot 10^{-4}$ \\
$C_{C_{l}}^{(Q)}$ & $9.9999 \cdot 10^{-1}$ & $9.9999 \cdot 10^{-1}$ & $9.9999 \cdot 10^{-1}$ & $9.9999 \cdot 10^{-1}$ \\
$C_{S}$ & $4.7890 \cdot 10^{-3}$ & $4.7916 \cdot 10^{-3}$ & $4.7932 \cdot 10^{-3}$ & $4.7938 \cdot 10^{-3}$ \\
\hline
\end{tabular}

Table 3: Amplitudes of the perturbations $\mathbf{u}_{\text {inf }}(\beta)$ for different values of $\beta$. The values shown correspond to normalised vectors.

and

$$
\mathbf{v}_{\text {inf }}(\beta)=\left(\begin{array}{c}
-\frac{1}{m_{s}} \\
\left.-\frac{k \mathcal{M}_{\beta}(\lambda ; \kappa \mathrm{Le})}{m_{s}\left(\mathcal{M}_{\beta}(\lambda ; \mathrm{Le})-\mathcal{U}_{\beta}(\lambda ; \mathrm{Le})\right.}\right) \\
\frac{k \mathcal{M}_{\beta}(\lambda ; \kappa \mathrm{Le})-\mathcal{M}_{\beta}(\lambda ; \mathrm{Le})}{m_{s}\left(\mathcal{M}_{\beta}(\lambda ; \mathrm{Le})-\mathcal{U}_{\beta}(\lambda ; \mathrm{Le})\right)} \\
1 \\
\frac{D m_{s}\left(\mathcal{M}_{\beta}(\lambda ; D)+\frac{2 \lambda}{D}\right)-m_{l}\left(\mathcal{U}_{\beta}(\lambda ; 1)+2 \lambda\right)}{m s\left(\mathcal{M}_{\beta}(\lambda ; 1)-\mathcal{U}_{\beta}(\lambda ; 1)\right)} \\
-\frac{D m_{s}\left(\mathcal{M}_{\beta}(\lambda ; D)+\frac{2 \lambda}{D}\right)-m_{l}\left(\mathcal{M}_{\beta}(\lambda ; 1)+2 \lambda\right)}{m s\left(\mathcal{M}_{\beta}(\lambda ; 1)-\mathcal{U}_{\beta}(\lambda ; 1)\right)} \\
0
\end{array}\right) .
$$

This choice of the vectors that generate the kernel is quite convenient as $\mathbf{v}_{\text {inf }}$ does not contribute to the perturbation of the interface (the last component is identically zero) and, because this is the only perturbation that will not decay in time for $-\frac{1}{2} \leqslant \beta<0$, we can conclude that the perturbations given by $\mathbf{v}_{\text {inf }}$ are stable for any $-\frac{1}{2} \leqslant \beta<0$ so the instability is determined by the perturbations given by $\mathbf{u}_{i n f}$. The other advantage of this kernel choice is that in $\mathbf{u}_{i n f}$ the amplitude of $c_{s 1}$ is zero, so the perturbations are similar to the ones in the semi-infinite domain. Even more, notice that if we take $c_{s 0}$ to be constant as in the semi-infinite domain (by taking $c_{s \infty}=\alpha c_{i}$ ) the vector $\mathbf{u}_{\text {inf }}$ reduces to $\mathbf{u}_{s e m i}$.

Remember that, as we stated in the semi-infinite domain analysis, we can show that the denominators of the elements of the kernel do not vanish for $\beta>-1$ so in our range of interest $-\frac{1}{2} \leqslant \beta<0$ the kernel is well defined. Similarly to the semi-infinite domain, the most unstable perturbation is when $\beta \rightarrow 0$ so its growth rate tends to the $t^{\frac{1}{2}}$ of the base state and we can find simpler forms for the vectors of the kernel.

In Figure 5 we show the perturbations given by $\mathbf{u}_{\text {inf }}$ for the same parameter choice as in the semi-infinite domain problem. We observe that their behaviour is very similar to the perturbations for the semi-infinite domain. In this case, temperature perturbations are negative if $\eta>0$ but $T_{s 1}$ is positive if $\eta<0$. This was not captured in the semi-infinite domain as we were restricted to $\eta \geqslant 0$. We still notice a spike of concetration just ahead of the interface and, again, the sign of the concentration of impurities in the liquid away from the interface is 


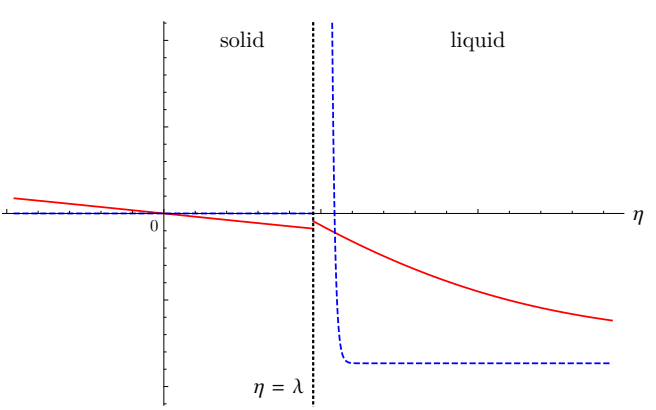

(a) $\beta=0$

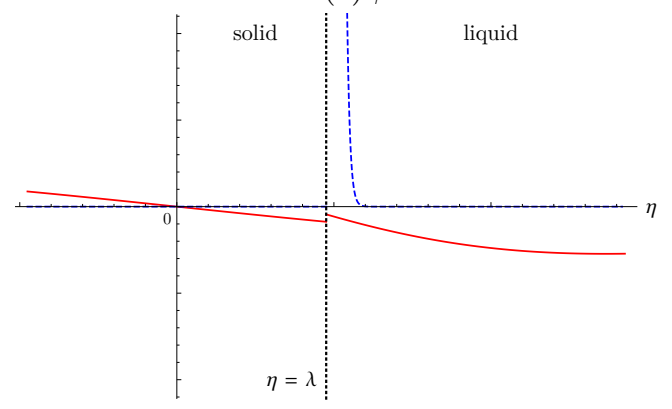

(c) $\beta=-\frac{1}{2}$

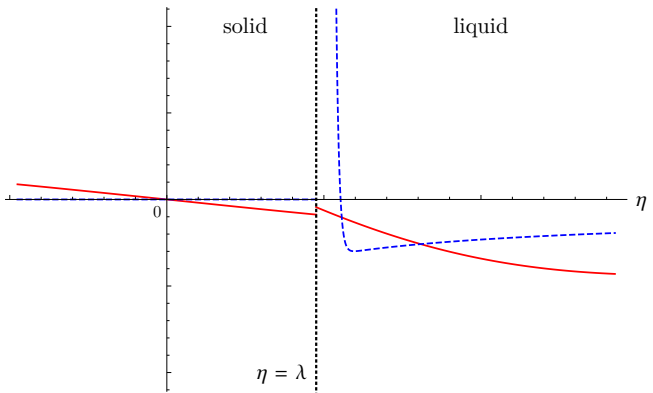

(b) $\beta=-\frac{1}{4}$

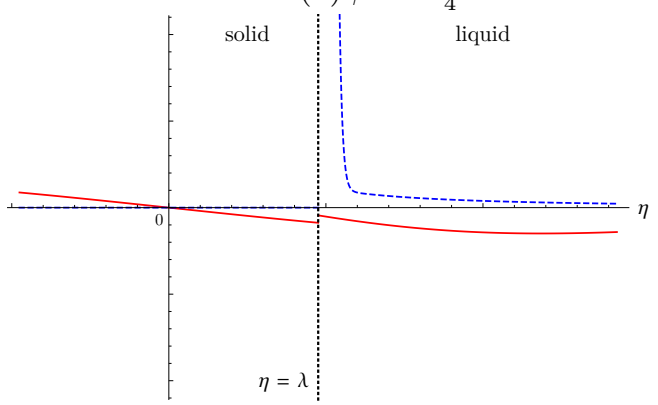

(d) $\beta=-\frac{3}{4}$

Fig. 5: Perturbations for the infinite domain for different values of $\beta$ using the amplitudes given by $\mathbf{u}_{\text {inf }}(\beta)$ (numerical values shown in Table 3 ). The domain of the perturbations is $\eta \in(-\infty,+\infty)$ as we are in the infinite domain problem defined by $z \in(-\infty,+\infty)$ and $t>0$. These perturbations introduce an instability because the amplitude of the perturbation of the interface $C_{S}$ is non-zero (not shown in the plots). Notice that, as it was pointed out from the form of $\mathbf{u}_{\text {inf }}$ these perturbations are very similar to the ones in Figure 4. The scaling is the same in all the plots so they are directly comparable. The parameters of the system have been set to $\mathrm{St}=0.77, \mathrm{Le}=134, \rho=0.95, c_{p}=1, k=2.91, \kappa=3.06, m_{s}=0.69, m_{l}=346$, $D=1.76 \cdot 10^{-2}, \alpha=2 \cdot 10^{-3}, T_{l \infty}=0$ and $c_{s \infty}=0$.

\begin{tabular}{|c||c|c|c|c|}
\hline$\beta$ & 0 & $-\frac{1}{4}$ & $-\frac{1}{2}$ & $-\frac{3}{4}$ \\
\hline \hline$C_{T_{s}}$ & $-2.9565 \cdot 10^{-3}$ & $-2.9271 \cdot 10^{-3}$ & $-2.8959 \cdot 10^{-3}$ & $-2.8629 \cdot 10^{-3}$ \\
$C_{T_{l}}^{(P)}$ & $-6.5120 \cdot 10^{-3}$ & $-6.9916 \cdot 10^{-3}$ & $-7.8645 \cdot 10^{-3}$ & $-1.0217 \cdot 10^{-2}$ \\
$C_{T_{l}}^{(Q)}$ & $3.5555 \cdot 10^{-3}$ & $4.0645 \cdot 10^{-3}$ & $4.9685 \cdot 10^{-3}$ & $7.3542 \cdot 10^{-3}$ \\
$C_{c_{s}}$ & $2.0400 \cdot 10^{-3}$ & $2.0197 \cdot 10^{-3}$ & $1.9982 \cdot 10^{-3}$ & $1.9754 \cdot 10^{-3}$ \\
$C_{c_{l}}^{(P)}$ & $2.3267 \cdot 10^{-2}$ & $1.2887 \cdot 10^{-2}$ & $2.0449 \cdot 10^{-3}$ & $-9.2943 \cdot 10^{-3}$ \\
$C_{\left.c_{l}\right)}^{(Q)}$ & $9.9970 \cdot 10^{-1}$ & $9.9988 \cdot 10^{-1}$ & $9.9995 \cdot 10^{-1}$ & $9.9987 \cdot 10^{-1}$ \\
$C_{S}$ & 0 & 0 & 0 & 0 \\
\hline
\end{tabular}

Table 4: Amplitudes of the perturbations $\mathbf{v}_{\text {inf }}(\beta)$ for different values of $\beta$. The values shown correspond to normalised vectors. 


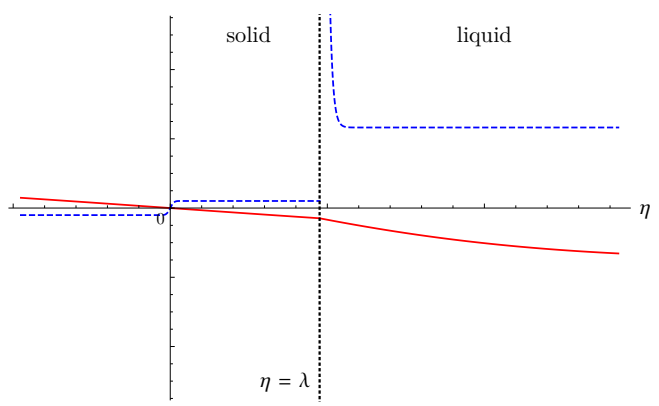

(a) $\beta=0$

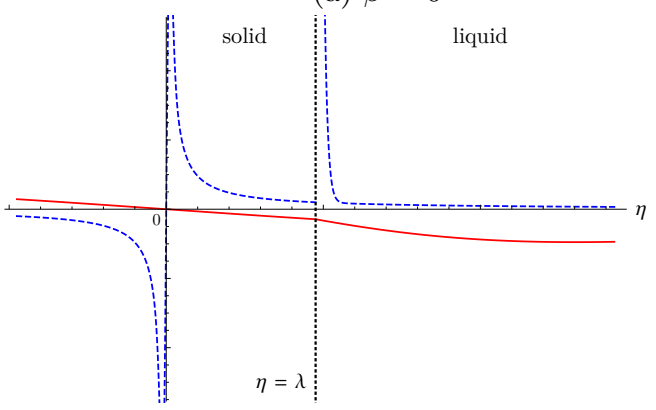

(c) $\beta=-\frac{1}{2}$

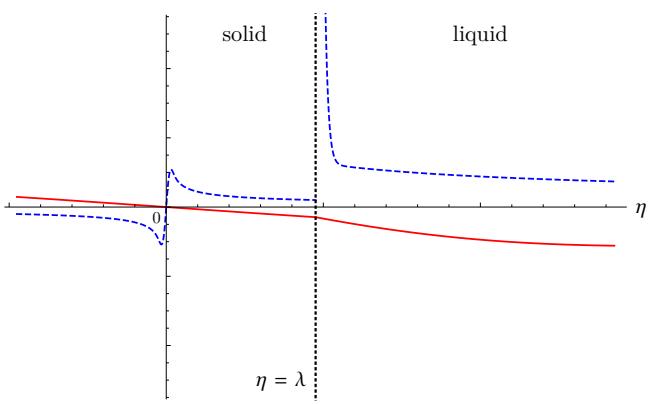

(b) $\beta=-\frac{1}{4}$

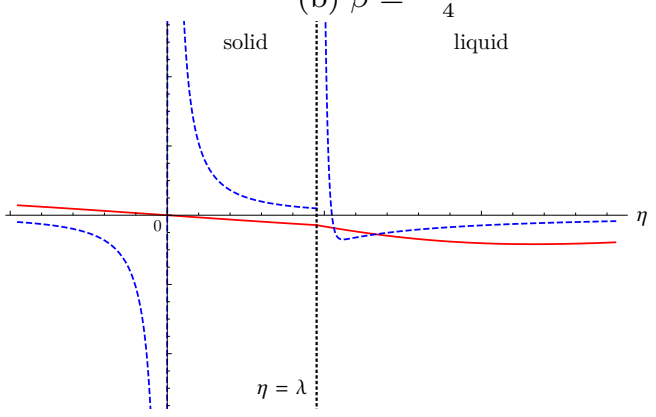

(d) $\beta=-\frac{3}{4}$

FIG. 6: Perturbations for the infinite domain for different values of $\beta$ using the amplitudes given by $\mathbf{v}_{\text {inf }}(\beta)$ (numerical values shown in Table 4). The domain of the perturbations is $\eta \in(-\infty,+\infty)$ as we are in the infinite domain problem defined by $z \in(-\infty,+\infty)$ and $t>0$. These perturbations do not introduce an instability, even if $-\frac{1}{2} \leqslant \beta<0$, because the amplitude of the perturbation of the interface $C_{S}$ is identically zero. The scaling is the same in all the plots so they are directly comparable. The parameters of the system have been set to St $=0.77$, Le $=134, \rho=0.95, c_{p}=1, k=2.91, \kappa=3.06, m_{s}=0.69, m_{l}=346, D=1.76 \cdot 10^{-2}$, $\alpha=2 \cdot 10^{-3}, T_{l \infty}=0$ and $c_{s \infty}=0$. 
negative in the unstable regime and positive in the stable regime. It quickly decays to zero if $\beta=-\frac{1}{2}$ as we can show from the fifth component of $\mathbf{u}_{\text {inf }}$ being identically zero.

In Figure 6 we show the perturbations given by $\mathbf{v}_{\text {inf }}$. In this case, the temperature perturbations are similar to the ones shown in the previous figures, but we notice qualitative changes in the concentration perturbations. The first change is that now we have a perturbation of the concentration in the solid. Bumps appear near the origin which become larger as $\beta$ decreases, however remember that these perturbations decay in time like $t^{\beta}$ so that the large bumps decay faster in time. The second thing we notice is that, even though the spike ahead of the interface is still present, far from the interface $c_{l 1}$ behaves differently when we change $\beta$. Now, $c_{l 1}$ is positive near $\beta=0$ and it becomes negative as we decrease $\beta$, but the sign change no longer happens at $\beta=-\frac{1}{2}$ as now the fifth component of $\mathbf{v}_{\text {inf }}$ does not vanish at $\beta=-\frac{1}{2}$.

The numerical values of the amplitudes used in the previous sketches are shown in Tables 3 and 4 . For the amplitudes given by $\mathbf{u}_{\text {inf }}(\beta)$ we observe a similar behaviour as in the amplitudes for $\mathbf{u}_{\text {semi }}(\beta)$ shown in Table 2. For the amplitudes given by $\mathbf{v}_{\text {inf }}(\beta)$ we notice that, again, the main contribution is $C_{c l}^{(Q)}$, even though these perturbations do not go unstable. We observe a very weak dependence of $C_{T_{s}}$ and $C_{c_{s}}$ on $\beta$ because this dependence is only introduced when normalising the vectors, as seen in the definition of $\mathbf{v}_{\text {inf }}(\beta)$

The analysis presented in this section is not only valid for solidification problems $(\lambda>0)$ but for melting problems as well $(\lambda<0)$. As the results would be very similar we do not show them here.

\section{Discussion}

We have considered the stability analysis for the self-similar motion of a planar solidification front for a binary alloy in two different geometries: the semi-infinite domain problem and the infinite domain problem. For both domains we introduce perturbations in the concentration and temperature fields of the form $t^{\beta} f(\eta)$, where $\beta$ is the parameter that determines the stability and $\eta$ is the similarity variable. Then the perturbation of the position of the interface is proportional to $t^{\beta+\frac{1}{2}}$, hence the exact base solution is locally unstable if $\beta>-\frac{1}{2}$. In each domain we need to separate the analysis into the cases $\beta \geqslant 0$ and $-\frac{1}{2} \leqslant \beta<0$. We find that in both domains, when $\beta \geqslant 0$, there are no non-trivial perturbations that satisfy the boundary conditions. Thus, we conclude that concentration and temperature fields are always stable, a result we could expect as the individual diffusion equations should give stable solutions in the interior of each sub-domain. Therefore, the instability in the exact solution is due to the growth of the perturbation to the interface.

Studying the case $-\frac{1}{2} \leqslant \beta<0$, we find instability in the self-similar solidification front for any parameter regime. In our analysis we have considered a more general type of perturbation than that considered in the analysis of Coriell et al. (1999), as we have kept both linearly independent first-order perturbation solutions. Keeping both solutions introduces a number of perturbations higher than the number of interface conditions available to determine them. Therefore, by the rank-nullity theorem, we can conclude that the kernel of the system will be of size one in the semi-infinite and size two in the infinite domain problem, thus never empty. For $-\frac{1}{2} \leqslant \beta<0$, the only perturbation that gives instability is the perturbation of the interface, but we have shown that we can always find a perturbation with a non-zero size perturbation of the interface. In conclusion, for both geometries we can always find an unstable perturbation so we conclude that, regardless of the parameter choice, the system is absolutely unstable. 
The main difference between the two geometries is that in the semi-infinite domain the kernel is of dimension one, while for the infinite domain problem the kernel is of dimension two so we find two families of perturbations. However, we can choose the elements of the kernels so that for one of them $C_{c_{s}}=0$ and for the other $C_{S}=0$. The advantage of this choice is that for the vector for which $C_{c_{s}}=0$ the perturbations are equivalent to the ones in the semi-infinite domain problem and for the vector for which $C_{S}=0$ the perturbations do not contribute to the instability of the problem. Therefore we find that even though the infinite domain geometry permits two types of perturbations, if we compare the unstable perturbation with the semi-infinite domain perturbations we see that their behaviour is very similar. Therefore, the mechanism bringing about instability is the same for both geometries.

We recall that Mullins and Sekerka (1964) found that the stability depended on the parameter choice of the system, with higher velocities and constitutional supercooling helping to destabilise the system. Our analysis shows that for a self-similar moving front the interface is always unstable regardless of the parameter choice. Therefore, it points that the cause of the instability may be intrinsic to the self-similar motion that appears when the cooling of molten silicon is not controlled.

Regarding possible future work, one hope is to extend these results to $2 \mathrm{D}$ domains. We expect that such an analysis would give us some insight into the grain size and will allow us to introduce two-dimensional physical effects, such as the Gibbs-Thomson effect, into the interface conditions. This will allow us to more realistically understand the loss of stability of the planar solidification front, and, perhaps, will provide a relation between grain size and the mode of instability.

The results presented here may be of use to the silicon industry, as they show that the instability of the planar interface is likely to occur for any parameter regime in solidification when the velocity of the interface is not controlled. Being able to understand these instabilities will possibly allow for more efficient casting techniques, as well as more control over the quality of the silicon produced.

\section{Acknowledgements}

This publication is based on work supported by the EPSRC Centre For Doctoral Training in Industrially Focused Mathematical Modelling (EP/L015803/1) in collaboration with Elkem AS. F. Brosa Planella is grateful to Elkem AS for the financial support and the opportunity to work in their site. We thank A. Autruffe, K. Hildal, and A. Valderhaug for useful discussions.

\section{References}

M. Abramowitz and I. A. Stegun, editors. Handbook of Mathematical Functions With Formulas, Graphs, and Mathematical Tables. U.S. Department of Commerce, NIST, 10 edition, 1964.

D. V. Alexandrov. The effect of concentrational supercooling on the morphological stability of self-similar solidification with a planar front. Doklady Physics, 46(7):453-458, 2001.

D. V. Alexandrov. Self-similar solidification: morphological stability of the regime. International Journal of Heat and Mass Transfer, 47(6):1383-1389, 2004.

J. C. Anglézio, C. Servant, and F. Dubrous. Characterization of metallurgical grade silicon. Journal of Materials Research, 5(09):1894-1899, 1990. 
G. P. Benham, K. Hildal, C. P. Please, and R. A. Van Gorder. Solidification of silicon in a one-dimensional slab and a two-dimensional wedge. International Journal of Heat and Mass Transfer, 98:530-540, 2016.

S. R. Coriell, G. B. McFadden, and R. F. Sekerka. Selection mechanisms for multiple similarity solutions for solidification and melting. Journal of Crystal Growth, 200(1):276-286, 1999.

R. A. Lambert and R. H. Rangel. Linear stability analysis of the solidification of a supercooled liquid in a half-space. International Journal of Heat and Mass Transfer, 45(23):4577-4587, 2002 .

J. S. Langer. Instabilities and pattern formation in crystal growth. Reviews of Modern Physics, 52(1):1-30, 1980.

M. F. Møll. Solidification of Silicon: Macro- and Microstructure as Functions of Thermal History and Composition. PhD thesis, NTNU Norges Teknisk-Naturvitenskapelige Universitet, 2014.

W. W. Mullins and R. F. Sekerka. Stability of a planar interface during solidification of a dilute binary alloy. Journal of Applied Physics, 35(2):444 - 451, 1964.

L. I. Rubinstein. The Stefan Problem, volume 27 of Translations of Mathematical Monographs. American Mathematical Society, 1971.

A. Schei, J. K. Tuset, and H. Tveit. Production of High Silicon Alloys. Tapir, 1998.

D. J. Wollkind and L. A. Segel. A nonlinear stability analysis of the freezing of a dilute binary alloy. Philosophical Transactions of the Royal Society of London A: Mathematical, Physical and Engineering Sciences, 268(1191):351 - 380, 1970.

M. G. Worster. Perspectives in Fluid Dynamics, chapter 8. Cambridge University Press, 2002. 